\title{
Sums of some multiplicative functions over a special set of integers
}

\author{
by \\ Y. K. LAU and J. Wu (Nancy)
}

1. Introduction. The sum of multiplicative functions and the value distribution of multiplicative functions are two central and important problems in analytic number theory. Two typical examples are

$$
\sum_{n \leq x} \mu(n) \text { and } \sum_{\varphi(n) \leq x} 1
$$

where $\mu(n)$ is the Möbius function and $\varphi(n)$ the Euler function. The first one is equivalent to the prime number theorem and the second was studied by Erdős \& Turán [5], Bateman [3] and Balazard \& Tenenbaum [2]. Another interesting aspect is the study of the value distribution restricted to a certain set of integers, for example, the set of squarefree integers [10] and the set of integers free of large prime factors [11]. It then seems natural to investigate a general sum

$$
F_{g}(x):=\sum_{g(n) \leq x} f(n),
$$

where $f(n)$ and $g(n)$ are two multiplicative functions. Problems of similar fashion have been considered by other authors ([1], [7], [9] and [10]). Generalizing a result of Abbott \& Subbarao, Balasubramanian \& Ramachandra [1] studied $\sum_{n g(n) \leq x} 1$ where $g$ is multiplicative, $g(p)$ equals a fixed positive constant for all primes $p$ and $g(n) \gg n^{-1 / 16}$ for all integers $n \geq 1$. By using tools of complex analysis, they established an asymptotic formula for this sum and answered a question of Erdős whether

$$
\sum_{n / \tau(n) \leq 2 x} 1 \sim 2 \sum_{n / \tau(n) \leq x} 1,
$$

where $\tau(n)$ is the divisor function. Using elementary methods, Smati [9]

2000 Mathematics Subject Classification: 11N37, 11L07. 
showed that there is a positive constant $c$ such that

$$
\sum_{g(n) \leq x} 1=A(g) x+O\left(x \exp \left\{-c \sqrt{\log x \log _{2} x}\right\}\right),
$$

where $A(g):=\prod_{p}\left(1-p^{-1}\right) \sum_{\nu=0}^{\infty} g\left(p^{\nu}\right)^{-1}, g$ is a multiplicative function and (i) $g\left(p^{\nu}\right)$ is a polynomial of degree $\nu$ in $p$ with leading coefficient 1 and all other coefficients in $[-1, B]$; (ii) $g(n) \gg n /\left(\log _{2} n\right)^{H}$ for all integers $n \geq 3$ ( $B \geq-1$ and $H>0$ are constants).

In this paper, we are concerned with the general sum $F_{g}(x)$ where both $f$ and $g$ are multiplicative and satisfy some conditions given below. Based on the Selberg-Delange method, we obtain an asymptotic formula for $F_{g}(x)$ with a very good error term. The error term is sharpest subject to the present techniques. Any improvement will lead to a better error term in the prime number theorem. In order to achieve this error term, we need to borrow the method of Balazard \& Tenenbaum [2]. Our result includes a wide class of multiplicative functions. Moreover, we can derive some new results related to local densities.

Let $\kappa, \theta, \theta^{\prime}, \theta^{\prime \prime}, \widetilde{\theta}, \alpha, \alpha^{\prime}, \eta, \psi, C_{1}, C_{2}, C_{3}$ be given constants such that

$$
\left\{\begin{array}{l}
|\kappa|<1 / \eta, \quad \theta>0, \quad \tilde{\theta}>\theta>\theta^{\prime}>\theta^{\prime \prime}, \quad \alpha>0, \quad \alpha^{\prime} \neq 0, \\
\eta>0, \quad \psi>1, \quad C_{1} \geq 0, \quad C_{2} \geq 0, \quad C_{3}>0 .
\end{array}\right.
$$

Suppose that $f: \mathbb{N} \rightarrow \mathbb{C}$ and $g: \mathbb{N} \rightarrow(0, \infty)$ are two multiplicative functions such that for all prime numbers $p$ :

(1) $|f(p)-\kappa| \leq C_{1} / p^{\eta}$;

(2) $g(p)=\alpha p^{\theta}$ or $g(p)=\alpha p^{\theta}+\alpha^{\prime} p^{\theta^{\prime}}+t(p)$, where $\left|t^{(l)}(u)\right| \leq\left(C_{2} l+1\right)^{l} u^{\theta^{\prime \prime}-l}(l=0,1, \ldots)$;

(3) $\sum_{\nu=2}^{\infty}\left|f\left(p^{\nu}\right)\right| / g\left(p^{\nu}\right)^{1 / \widetilde{\theta}} \leq C_{3} / p^{\psi}$.

As usual let $\tau_{\kappa}(n)$ be the Piltz function, defined by

$$
\zeta(s)^{\kappa}=\sum_{n=1}^{\infty} \tau_{\kappa}(n) / n^{s},
$$

where $\zeta(s)$ is the Riemann function. In particular $\tau_{2}(n)=\tau(n)$ is the usual divisor function. Define

$$
\mathbf{1}(n) \equiv 1, \quad j(n):=n, \quad \sigma(n):=\sum_{d \mid n} d, \quad \Omega(n):=\sum_{p^{\nu} \| n} \nu, \quad \omega(n):=\sum_{p^{\nu} \| n} 1 .
$$

It is easy to verify that the following function pairs:

$$
(\mu, j), \quad(\mathbf{1}, \varphi), \quad(\mu, \varphi), \quad(\mathbf{1}, j / \tau), \quad\left(\mu^{2}, \sigma\right), \quad\left(\tau_{\kappa}, j\right), \quad\left(z^{\Omega}, j\right), \quad\left(z^{\omega}, \varphi\right)
$$

satisfy our assumptions on $(f, g)$ for suitable parameters. 
For the Selberg-Delange method, we introduce the associated Dirichlet series

$$
\mathcal{F}_{g}(s):=\sum_{n=1}^{\infty} \frac{f(n)}{g(n)^{s}}, \quad \widetilde{\mathcal{F}}_{g}(s):=\frac{\mathcal{F}_{g}(s)}{\zeta(\theta s)^{\kappa / \alpha^{s}}} .
$$

Under our assumptions (1)-(3), it is not difficult to show that there is a positive constant $\varrho_{0}$ such that $\widetilde{\mathcal{F}}_{g}(s)$ is uniformly convergent on any compact set in the half-plane $\sigma \geq 1 / \theta-10 \varrho_{0}$ (see Theorem 4(i)). Thus we can write, for $|s-1 / \theta|<10 \varrho_{0}$,

$$
s^{-1} \widetilde{\mathcal{F}}_{g}(s)(\zeta(\theta s)(\theta s-1))^{\kappa / \alpha^{s}}=\sum_{l=0}^{\infty} a_{l}(s-1 / \theta)^{l}=\sum_{l=0}^{\infty} \frac{a_{l}}{\theta^{l}}(\theta s-1)^{l},
$$

where the coefficient $a_{l}=a_{l}(f, g)$ is given by

$$
a_{l}:=\frac{1}{2 \pi i} \int_{|s-1 / \theta|=\varrho_{0}} \frac{\widetilde{\mathcal{F}}_{g}(s)(\zeta(s)(s-1))^{\kappa / \alpha^{s}}}{s(s-1 / \theta)^{l+1}} d s \ll \frac{1}{\varrho_{0}^{l}} .
$$

In particular

$$
a_{0}=\prod_{p}(1-1 / p)^{\kappa / \alpha^{1 / \theta}} \sum_{\nu=0}^{\infty} f\left(p^{\nu}\right) / g\left(p^{\nu}\right)^{1 / \theta} .
$$

Let

$$
\begin{aligned}
b_{m, n} & :=\sum_{n_{1}+\ldots+n_{m}=n} \frac{1}{\left(n_{1}+1\right) ! \ldots\left(n_{m}+1\right) !} \quad(m \geq 1), \\
b_{0, n} & := \begin{cases}1 & \text { if } n=0 \\
0 & \text { if } n \geq 1 .\end{cases}
\end{aligned}
$$

As usual we use $\Gamma(s)$ to denote the Euler $\Gamma$-function and define

$$
\frac{1}{\Gamma_{i}(a)}:=\left[\frac{d^{i}}{d z^{i}}\left(\frac{1}{\Gamma(z)}\right)\right]_{z=a} .
$$

Put $\mathcal{L}(x):=\exp \left\{(\log x)^{3 / 5} /\left(\log _{2} x\right)^{1 / 5}\right\}$, where $\log _{k}$ is the $k$-fold iterated logarithm. Let $c_{i}$ and $\varrho_{i}$ be constants depending at most on $\theta, \theta^{\prime}, \theta^{\prime \prime}, \widetilde{\theta}, \alpha$, $\alpha^{\prime}, \eta, \psi, \psi_{1}, \psi_{2}, C_{1}, C_{2}, C_{3}, C_{4}$ (see Theorem 2 below). The $\varrho_{i}$ are small enough to satisfy the conditions made precise later. The constants implied in $\ll$ or $O$ depend at most on these parameters. Finally we define $0^{0}=1$.

THEOREM 1. Let $f, g$ satisfy the assumptions (1)-(3). Then for any integer $J \geq 0$, we have

$$
F_{g}(x)=\frac{x^{1 / \theta}}{(\log x)^{1-\kappa / \alpha^{1 / \theta}}}\left\{\sum_{j=0}^{J} \frac{P_{j}\left(\log _{2} x\right)}{(\log x)^{j}}+O\left(R_{J, \lambda}(x)\right)\right\},
$$


where $P_{j}(t):=\sum_{l=0}^{j} \lambda_{j, l} t^{l}$ and the coefficient $\lambda_{j, l}=\lambda_{j, l}(f, g)$ is given by

$$
\lambda_{j, l}:=\frac{\theta^{-\kappa / \alpha^{1 / \theta}}}{l !} \sum_{m=l}^{j} \sum_{k=l}^{m} \sum_{i=0}^{k-l} \lambda_{m, k, i}^{*}
$$

where

$$
\lambda_{m, k, i}^{*}:=\frac{(-\log \alpha)^{m}\left(\kappa / \alpha^{1 / \theta}\right)^{k}(-\log \theta)^{k-l-i} a_{j-m} b_{k, m-k}}{(k-l-i) ! i ! \Gamma_{i}\left(\kappa / \alpha^{1 / \theta}-j\right)} .
$$

The error term $R_{J, \lambda}(x)$ is defined by

$$
R_{J, \lambda}(x):=\left(\left(c_{1} J+1\right) \frac{\lambda \log _{2} x+c_{2}}{\log x}\right)^{J+1}+\frac{1}{\mathcal{L}(x)^{c_{3}}}
$$

with $\lambda:=\varrho_{0} \alpha^{-1 / \theta}|\kappa \log \alpha| e^{\varrho_{0}|\log \alpha|}<1$. Moreover,

$$
\lambda_{j, l} \ll \lambda^{l}\left(c_{4} j+1\right)^{j} / l ! .
$$

Remarks. (i) The dominating term in the sum (1.6) is given by

$$
\frac{a_{0}}{\theta^{\kappa / \alpha^{1 / \theta}} \Gamma\left(\kappa / \alpha^{1 / \theta}\right)}
$$

if $a_{0} / \theta^{\kappa / \alpha^{1 / \theta}} \Gamma\left(\kappa / \alpha^{1 / \theta}\right) \neq 0$. This is true when $\sum_{\nu=0}^{\infty} f\left(p^{\nu}\right) / g\left(p^{\nu}\right)^{1 / \theta} \neq 0$ for all prime numbers $p$ (see (1.5)) and $\kappa / \alpha^{1 / \theta} \neq 0,-1,-2, \ldots$

(ii) If $\alpha=1$, then $\lambda=0$ and $\lambda_{j, l}=0$ for $l=1, \ldots, j$. Thus the asymptotic formula (1.6) in Theorem 1 is simplified to

$$
F_{g}(x)=\frac{x^{1 / \theta}}{(\log x)^{1-\kappa}}\left\{\sum_{j=0}^{J} \frac{\lambda_{j, 0}}{(\log x)^{j}}+O\left(R_{J}(x)\right)\right\},
$$

where $\lambda_{j, 0}=a_{j} / \theta^{\kappa} \Gamma(\kappa-j)$ and

$$
R_{J}(x):=\left(\frac{J+1}{c_{5} \log x}\right)^{J+1}+\frac{1}{\mathcal{L}(x)^{c_{3}}} .
$$

(iii) If $\alpha=1$ and $\kappa=J_{0} \in \mathbb{Z}$, then $\lambda=0, \lambda_{j, l}=0$ for $l=1, \ldots, j$ and $\lambda_{j, 0}=0$ for $j \geq J_{0}$. Taking $J=(\log x)^{3 / 5} /\left(\log _{2} x\right)^{6 / 5}$ in (1.10), we obtain

$$
F_{g}(x)=\frac{x^{1 / \theta}}{(\log x)^{1-J_{0}}}\left\{\sum_{j=0}^{J_{0}-1} \frac{\lambda_{j, 0}}{(\log x)^{j}}+O\left(\mathcal{L}(x)^{-c_{6}}\right)\right\} .
$$

In particular if $J_{0} \leq 0$, then

$$
F_{g}(x) \ll x^{1 / \theta} / \mathcal{L}(x)^{c_{7}} .
$$

The next result comes from particular cases of (1.12) and (1.13). The first assertion is due to Balazard \& Tenenbaum ([2], théorème 1), the second one is equivalent to the prime number theorem and the third one is new. 
Corollary 1. There is a constant $c>0$ such that

(i) $\sum_{\varphi(n) \leq x} 1=\{\zeta(2) \zeta(3) / \zeta(6)\} x+O\left(x / \mathcal{L}(x)^{c}\right)$;

(ii) $\sum_{n \leq x} \mu(n) \ll x / \mathcal{L}(x)^{c}$;

(iii) $\sum_{\varphi(n) \leq x} \mu(n) \ll x / \mathcal{L}(x)^{c}$.

Clearly Theorem 1 also improves Smati's (1.1), Corollaries 1-4 of Scourfield in [7], contains Selberg's asymptotic formula for $\sum_{n \leq x} \tau_{\kappa}(n)$ [8] and generalizes the main result of Balasubramanian \& Ramachandra [1].

From Theorem 1, we can derive some local density results.

THEOREM 2. Let $f$ and $g$ satisfy the assumptions (1), (2) and furthermore assume

$(3)^{\prime}\left|f\left(p^{\nu}\right)\right| \leq C_{4} g\left(p^{\nu}\right)^{1 / \widetilde{\theta}}\left(\psi_{1} / p\right)^{\psi_{2} \nu}$ for all primes $p$ and all integers $\nu \geq 2$, where $\widetilde{\theta}>\theta, \psi_{1}>1 / 2$ and $\psi_{2}>1 / 2$ are given constants.

Let $\lambda$ and $R_{J, \lambda}(x)$ be defined as in Theorem 1 . Then for any integer $J \geq 0$ and any $\varepsilon>0$, we have, uniformly for $x \geq 3$ and $1 \leq k \leq$ $\left((2-\varepsilon) / \psi_{1}\right)^{\psi_{2}} \log _{2} x$,

$$
\begin{aligned}
\sum_{\substack{g(n) \leq x \\
\Omega(n)=k}} f(n)= & \frac{x^{1 / \theta}}{\log x}\left\{\sum_{j=0}^{J} \frac{Q_{j, k}\left(\log _{2} x\right)}{(\log x)^{j}}\right. \\
& \left.+O\left(\left(\frac{\log _{2} x}{k}\right)^{k} \frac{e^{k|\kappa| \alpha^{-1 / \theta}}}{\sqrt{|\varrho| k+1}} R_{J, \lambda}(x)\right)\right\},
\end{aligned}
$$

where $Q_{j, k}(t):=\sum_{n=0}^{j+k-1} \lambda_{j, n, k} t^{n}$ and the coefficient $\lambda_{j, n, k}$ is given by

$$
\lambda_{j, n, k}:=\sum_{\substack{l+m=n \\ 0 \leq l \leq j, 0 \leq m \leq k}} \frac{\left(\kappa \alpha^{-1 / \theta}\right)^{m}}{2 \pi i m !} \oint_{|z|=1} \frac{\lambda_{j, l}\left(f z^{\Omega}, g\right)}{z^{k+1-m}} d z
$$

and $\lambda_{j, l}\left(f z^{\Omega}, g\right)$ is defined by (1.7).

Theorem 3. Let $f, g, \lambda$ and $R_{J, \lambda}(x)$ be defined as in Theorem 1 . Then for any $A>0$ and any integer $J \geq 0$, we have, uniformly for $x \geq 3$ and $1 \leq k \leq A \log _{2} x$

$$
\begin{aligned}
\sum_{\substack{g(n) \leq x \\
\omega(n)=k}} f(n)= & \frac{x^{1 / \theta}}{\log x}\left\{\sum_{j=0}^{J} \frac{\widetilde{Q}_{j, k}\left(\log _{2} x\right)}{(\log x)^{j}}\right. \\
& \left.+O_{A}\left(\left(\frac{\log _{2} x}{k}\right)^{k} \frac{e^{k|\kappa| \alpha^{-1 / \theta}}}{\sqrt{|\kappa| k+1}} R_{J, \lambda}(x)\right)\right\},
\end{aligned}
$$


where $\widetilde{Q}_{j, k}(t):=\sum_{n=0}^{j+k-1} \widetilde{\lambda}_{j, n, k} t^{n}$ and the coefficient $\widetilde{\lambda}_{j, n, k}$ is given by

$$
\widetilde{\lambda}_{j, n, k}:=\sum_{\substack{l+m=n \\ 0 \leq l \leq j, 0 \leq m \leq k}} \frac{\left(\kappa \alpha^{-1 / \theta}\right)^{m}}{2 \pi i m !} \oint_{|z|=1} \frac{\lambda_{j, l}\left(f z^{\omega}, g\right)}{z^{k+1-m}} d z
$$

and $\lambda_{j, l}\left(f z^{\omega}, g\right)$ is defined by (1.7).

Clearly Theorems 2 and 3 contain Selberg's classical results [8] on $N_{k}(x):=|\{n \leq x: \Omega(n)=k\}| \quad$ and $\quad \pi_{k}(x):=|\{n \leq x: \omega(n)=k\}|$.

Here we state some consequences, which are new.

Corollary 2. For any $\delta>0$, we have, uniformly for $J \geq 0, x \geq 3$ and $1 \leq k \leq(2-\delta) \log _{2} x$,

$$
\sum_{\substack{\varphi(n) \leq x \\ \Omega(n)=k}} 1=\frac{x}{\log x}\left\{\sum_{j=0}^{J} \frac{W_{j, k}\left(\log _{2} x\right)}{(\log x)^{j}}+O_{\delta}\left(\frac{\left(\log _{2} x\right)^{k}}{k !} R_{J}(x)\right)\right\},
$$

where

$$
W_{j, k}(t):=\sum_{n=0}^{k-1} \frac{\varsigma_{j}^{(k-1-n)}(0)}{n !(k-1-n) !} t^{n}, \quad \varsigma_{j}(z):=\frac{a_{j}\left(z^{\Omega}, \varphi\right)}{z \Gamma(z-j)}
$$

and $a_{j}\left(z^{\Omega}, \varphi\right)$ is defined by (1.4). Moreover, under the same conditions,

$$
\sum_{\substack{\varphi(n) \leq x \\ \Omega(n)=k}} 1=\frac{x}{\log x} \cdot \frac{\left(\log _{2} x\right)^{k-1}}{(k-1) !}\left\{\varsigma_{0}\left(\frac{k-1}{\log _{2} x}\right)+O_{\delta}\left(\frac{k}{\left(\log _{2} x\right)^{2}}\right)\right\},
$$

where

$$
\varsigma_{0}(z)=\frac{1}{\Gamma(z+1)} \prod_{p}\left(1+\frac{p z}{(p-1)(p-z)}\right)\left(1-\frac{1}{p}\right)^{z} .
$$

Corollary 3. For any $A>0$, we have, uniformly for $J \geq 0, x \geq 3$ and $1 \leq k \leq A \log _{2} x$,

$$
\sum_{\substack{\varphi(n) \leq x \\ \omega(n)=k}} 1=\frac{x}{\log x}\left\{\sum_{j=0}^{J} \frac{\widetilde{W}_{j, k}\left(\log _{2} x\right)}{(\log x)^{j}}+O_{A}\left(\frac{\left(\log _{2} x\right)^{k}}{k !} R_{J}(x)\right)\right\}
$$

where

$$
\widetilde{W}_{j, k}(t):=\sum_{n=0}^{k-1} \frac{\widetilde{\varsigma}_{j}^{(k-1-n)}(0)}{n !(k-1-n) !} t^{n}, \quad \widetilde{\varsigma}_{j}(z):=\frac{a_{j}\left(z^{\omega}, \varphi\right)}{z \Gamma(z-j)}
$$


and $a_{j}\left(z^{\omega}, \varphi\right)$ is defined by (1.4). Moreover, under the same conditions,

$$
\sum_{\substack{\varphi(n) \leq x \\ \omega(n)=k}} 1=\frac{x}{\log x} \cdot \frac{\left(\log _{2} x\right)^{k-1}}{(k-1) !}\left\{\widetilde{\varsigma}_{0}\left(\frac{k-1}{\log _{2} x}\right)+O_{A}\left(\frac{k}{\left(\log _{2} x\right)^{2}}\right)\right\},
$$

where

$$
\widetilde{\varsigma}_{0}(z)=\frac{1}{\Gamma(z+1)} \prod_{p}\left(1+\frac{p z}{(p-1)^{2}}\right)\left(1-\frac{1}{p}\right)^{z} .
$$

In what follows the letter $s$ always denotes a complex number and we implicitly define the real numbers $\sigma$ and $\tau$ by the relation $s=\sigma+i \tau$. We let $\beta(t):=(\log t)^{-2 / 3}\left(\log _{2} t\right)^{-1 / 3}$ for $t \geq 3$ and $T:=|\tau|+3$. The next result is a generalization of Theorem 2 in [2], which plays a key role in the proof of Theorem 1.

THEOREM 4. Under the above conditions, there is a positive constant $\varrho_{0}$ such that

(i) $\widetilde{\mathcal{F}}_{g}(s)$ is uniformly convergent on any compact set in the half-plane $\sigma \geq 1 / \theta-10 \varrho_{0}$

(ii) $\left|\widetilde{\mathcal{F}}_{g}(s)\right| \ll\left(\log ^{2} T \log _{2} T\right)^{\frac{2}{3}|\kappa| \alpha^{-\sigma}}$ for $\sigma \geq 1 / \theta-10 \varrho_{0} \beta(T)$.

Acknowledgements. The authors take pleasure in thanking G. Tenenbaum for his help during the preparation of this paper. The first named author is supported by a postdoctoral fellowship of the French Ministry of Education, Research and Technology. As well, he would express his gratitude for the hospitality at Institut Élie Cartan.

2. Some preparations. This section is devoted to proving some preliminary lemmas. The following result is a variant of Lemma 1 of [2].

LEMma 1. Let $0<b_{0}<1<b_{1}, \delta>0$ be fixed and $\delta_{0}:=\min \{\sqrt{\delta} / 4,1 / 10\}$. Let $P \in \mathbb{Z}, Q \in \mathbb{N}$ and $D \geq Q^{\delta}$. Suppose that $h \in \mathcal{C}^{\infty}([P, P+Q], \mathbb{R})$ and for all $P \leq u \leq P+Q$ and $1 \leq l \leq \log D /\left(8 \delta_{0}^{2} \log Q\right)+1$,

$$
D /\left(b_{1} Q\right)^{l} \leq\left|h^{(l)}(u)\right| / l ! \leq D /\left(b_{0} Q\right)^{l} .
$$

Then there exist three positive constants $A=A(\delta), c=c(\delta)$ and $Q_{0}=$ $\max \left\{b_{0}^{-1 / \delta_{0}^{2}}, b_{1}^{1 / \delta_{0}}\right\}$ such that

$$
\max _{Q_{1} \leq Q}\left|\sum_{P<n \leq P+Q_{1}} e(h(n))\right| \leq A Q \exp \left\{-c(\log Q)^{3} /(\log D)^{2}\right\} \quad\left(Q \geq Q_{0}\right),
$$

where $e(t)=e^{2 \pi i t}(t \in \mathbb{R})$. 
Proof. For $Q \geq Q_{0}$, we choose $K \in \mathbb{N}$ such that $Q^{8 \delta_{0}^{2}(K-1)} \leq D<$ $Q^{8 \delta_{0}^{2} K}$. Then

$$
\begin{aligned}
& 2 \leq \log D /\left(8 \delta_{0}^{2} \log Q\right)<K \leq \log D /\left(8 \delta_{0}^{2} \log Q\right)+1, \\
& D /\left(b_{0} Q\right)^{K} \leq Q^{-\left(1-9 \delta_{0}^{2}\right) K} /\left(b_{0} Q^{\delta_{0}^{2}}\right)^{K} \leq Q^{-\left(1-9 \delta_{0}^{2}\right) K} .
\end{aligned}
$$

Similarly for $\delta_{0} K \leq l \leq 2 \delta_{0} K$, we have

$$
\begin{aligned}
D /\left(b_{0} Q\right)^{l} & \leq Q^{8 \delta_{0}^{2} K} /\left(b_{0} Q\right)^{l} \leq Q^{-\left(1-9 \delta_{0}\right) l} /\left(b_{0} Q^{\delta_{0}}\right)^{l} \leq Q^{-\left(1-9 \delta_{0}\right) l}, \\
D /\left(b_{1} Q\right)^{l} & \geq Q^{8 \delta_{0}^{2}(K-1)} /\left(b_{1} Q\right)^{l} \geq Q^{4 \delta_{0}^{2} K} /\left(b_{1} Q\right)^{l} \\
& \geq Q^{-\left(1-\delta_{0}\right) l}\left(Q^{\delta_{0}} / b_{1}\right)^{l} \geq Q^{-\left(1-\delta_{0}\right) l} .
\end{aligned}
$$

Our result follows immediately by Lemma 0 in [2].

Let $\Lambda(n)$ be the von Mangoldt function and $g_{0}(u):=\alpha u^{\theta}+\alpha^{\prime} u^{\theta^{\prime}}+t(u)$. Define

$$
S_{M}(\tau):=\sup _{M<N \leq 2 M}\left|\sum_{M<n \leq N} \Lambda(n) g_{0}(n)^{-i \tau}\right| .
$$

The next two lemmas are generalizations of Lemmas 2 and 3 in [2], which play a key role in the proof of Theorem 4.

Lemma 2. There exists a positive constant $\varrho_{1}$ such that

$$
S_{M}(\tau) \ll M^{1-\varrho_{1} \beta(T)}+M T^{-1}+M \mathcal{L}(M)^{-\varrho_{1}}
$$

for $T:=|\tau|+3 \leq M^{\theta_{1}\left(1+\varrho_{1} \beta(M)\right)}$, where $\theta_{1}:=\theta-\theta^{\prime}$.

Proof. Define

$$
V_{\tau}(z):=\sum_{n \leq z} \frac{\Lambda(n)}{n^{i \tau}}, \quad w_{\tau}(z):=\frac{z^{i \theta \tau}}{g_{0}(z)^{i \tau} \log z} .
$$

It is easy to see that

$$
\frac{d w_{\tau}(z)}{d z} \ll \frac{z^{-\theta_{1}} T+1}{z \log z} .
$$

Thus we can deduce that for $M \leq N \leq 2 M$,

$$
\begin{aligned}
\sum_{M<p \leq N} g_{0}(p)^{-i \tau} & =\sum_{M<n \leq N} w_{\tau}(n) \frac{\Lambda(n)}{n^{i \theta \tau}}+O(1) \\
& =\int_{M}^{N} w_{\tau}(z) d V_{\theta \tau}(z)+O(1) \\
& \ll \sup _{M \leq z \leq N}\left|V_{\theta \tau}(z)\right|\left(\frac{1}{\log M}+\int_{M}^{N} \frac{z^{-\theta_{1}} T+1}{z \log z} d z\right)+1 \\
& \ll \sup _{M \leq z \leq N}\left|V_{\theta \tau}(z)\right|\left(M^{-\theta_{1}} T+1\right)+1 .
\end{aligned}
$$


According to (12) in [2], we have $V_{\tau}(z) \ll z^{1-c \beta(T)}+z T^{-1}+z \mathcal{L}(z)^{-c}$ for $z \geq 3$ and $\log T \leq(\log z)^{3 / 2} /\left(\log _{2} z\right)^{2}$, where $c>0$ is an absolute constant. Hence we deduce that for some suitable constant $c_{8}>0$,

$$
\begin{aligned}
\sum_{M<p \leq N} g_{0}(p)^{-i \tau} & \ll\left(M^{-\theta_{1}} T+1\right)\left(M^{1-c_{8} \beta(T)}+M / T+M / \mathcal{L}(M)^{c_{8}}\right) \\
& \ll M^{1-c_{8} \beta(T)+\theta_{1} \varrho_{1} \beta(M)}+M / T+M^{1+\theta_{1} \varrho_{1} \beta(M)} / \mathcal{L}(M)^{c_{8}},
\end{aligned}
$$

which implies the desired result provided $\varrho_{1}>0$ is suitably small.

Lemma 3. There exist two positive constants $\varrho_{2}$ and $\varrho_{3}$ such that

$$
S_{M}(\tau) \ll M\left\{e^{-\varrho_{2}(\log M)^{3} /(\log T)^{2}}+\left(M^{\theta_{1}} / T\right)^{1 / 2}(\log M)^{7 / 2}\right\}
$$

for $3 \leq M^{\theta_{1}} \leq T:=|\tau|+3 \leq \exp \left\{\varrho_{3}(\log M)^{2}\right\}$, where $\theta_{1}:=\theta-\theta^{\prime}$.

Proof. Clearly the assertion is trivial if $T \leq 10$. Thus we can suppose that $T>10$ and $T \asymp|\tau|$. We define $\theta_{2}:=\theta-\theta^{\prime \prime}, \theta_{0}:=\min \left\{3 \theta_{1} / 2, \theta_{2}\right\}$ and

$$
\delta:=\min \left\{\frac{\theta_{0}-\theta_{1}}{2\left(3+\theta_{0}+\theta_{1}\right)}, \frac{1}{12\left(1+\theta_{1}\right)}\right\}>0 .
$$

Applying Vaughan's identity ([4], (24.6)) with $U=V=M^{1 / 2-\delta}$, for $M \leq$ $N \leq 2 M$ we have

$$
\begin{aligned}
\sum_{n \leq N} \Lambda(n) g_{0}(n)^{-i \tau} & \\
& \ll M^{1 / 2-\delta}+S_{N}^{\prime}(\tau) \log M+\left\{S_{N}^{\prime \prime}(\tau) M\right\}^{1 / 2}(\log M)^{3},
\end{aligned}
$$

where

$$
\begin{aligned}
& S_{N}^{\prime}(\tau):=\sum_{n \leq M^{1-2 \delta}} \max _{w}\left|\sum_{w \leq r \leq N / n} g_{0}(n r)^{-i \tau}\right|, \\
& S_{N}^{\prime \prime}(\tau):=\max _{\substack{M^{1 / 2-\delta} \leq Q \leq N / M^{1 / 2-\delta} \\
M^{1 / 2-\delta}<j \leq N / Q}} \sum_{M^{1 / 2-\delta}<k \leq N / Q}\left|\sum_{\substack{Q<m \leq 2 Q \\
m \leq N / \max \{j, k\}}}\left(\frac{g_{0}(k m)}{g_{0}(j m)}\right)^{i \tau}\right| .
\end{aligned}
$$

In order to bound the sum $S_{N}^{\prime}(\tau)$, we first observe that

$$
\begin{aligned}
& S_{N}^{\prime}(\tau) \leq M^{1-\delta} \\
& \quad+\sum_{n \leq M^{1-2 \delta}} \max _{M^{\delta} \leq w \leq N / n} \sum_{\nu \geq 0}\left|\sum_{\max \left\{w, N /\left(2^{\nu+1} n\right)\right\} \leq r \leq N /\left(2^{\nu} n\right)} g_{0}(n r)^{-i \tau}\right| .
\end{aligned}
$$

Thus it is sufficient to bound the sum $\sum_{U<r \leq U^{\prime}} e(h(r))$, where $h(u):=$ $-(\tau /(2 \pi)) \log g_{0}(n u), M^{\delta} \leq U \leq \min \{N / n, M\}$ and $U+M^{\delta} \leq U^{\prime} \leq 2 U$. This will be done by applying Lemma 1 . Let $v(u):=\alpha^{\prime} u^{\theta^{\prime}}+t(u)$. We expand 
$h(u)$ in a series form

$$
h(u)=-\frac{\tau}{2 \pi}\left\{\log \alpha+\theta \log (n u)-\sum_{\nu=1}^{\infty} \frac{(-1)^{\nu}}{\nu \alpha^{\nu}}\left(\frac{v(n u)}{(n u)^{\theta}}\right)^{\nu}\right\} .
$$

By the assumption (2), we easily show that $\left|v^{(l)}(u)\right| \leq\left(c_{9} l+1\right)^{l} u^{\theta^{\prime}-l}$. Note that

$$
\frac{d^{l}}{d u^{l}} \phi(n u)=n^{l} \phi^{(l)}(n u), \quad \frac{d^{l}}{d u^{l}} \prod_{i=1}^{\nu} \phi_{i}(u)=\sum_{l_{1}+\ldots+l_{\nu}=l} \frac{l !}{l_{1} ! \ldots l_{\nu} !} \prod_{i=1}^{\nu} \phi_{i}^{\left(l_{i}\right)}(u) .
$$

Then for $l \geq 1$ we have

$$
\begin{aligned}
& \left|\frac{d^{l}}{d u^{l}}\left(\frac{v(n u)}{(n u)^{\theta}}\right)^{\nu}\right| \\
& =n^{l}\left|\sum_{l_{1}+\ldots+l_{\nu+1}=l} \frac{(-1)^{l_{\nu+1}} l !(\theta \nu) \ldots\left(\theta \nu+l_{\nu+1}-1\right)}{l_{1} ! \ldots l_{\nu+1} !(n u)^{\theta \nu+l_{\nu+1}}} \prod_{i=1}^{\nu} v^{\left(l_{i}\right)}(n u)\right| \\
& \leq \frac{l !}{(n u)^{\theta_{1} \nu} u^{l}} \sum_{l_{1}+\ldots+l_{\nu+1}=l} \frac{(\theta \nu) \ldots\left(\theta \nu+l_{\nu+1}-1\right)}{l_{1} ! \ldots l_{\nu+1} !} \prod_{i=1}^{\nu}\left(c_{9} l_{i}+1\right)^{l_{i}} \\
& \leq \frac{l !}{(n u)^{\theta_{1} \nu} u^{l}} \sum_{l_{1}+\ldots+l_{\nu+1}=l} \frac{\left(c_{10} l\right)^{l_{1}+\ldots+l_{\nu}}(\theta \nu+l)^{l_{\nu+1}}}{l_{1} ! \ldots l_{\nu+1} !} \\
& \leq\left(c_{11} \nu l\right)^{l}(n u)^{-\theta_{1} \nu} u^{-l} .
\end{aligned}
$$

Hence if $1 \leq l \leq c_{12} \log U$ and $U \leq u \leq 2 U$, we deduce that

$$
\begin{aligned}
\frac{d^{l}}{d u^{l}} \sum_{\nu=1}^{\infty} \frac{(-1)^{\nu}}{\nu \alpha^{\nu}}\left(\frac{v(n u)}{(n u)^{\theta}}\right)^{\nu} & \leq\left(\frac{c_{11} l}{u}\right)^{l} \sum_{\nu=1}^{\infty} \frac{\nu^{l-1}}{\left(\alpha(n u)^{\theta_{1}}\right)^{\nu}} \\
& \leq\left(\frac{c_{13} l}{u}\right)^{l} \sum_{\nu=1}^{\infty} \frac{1}{\left(\alpha(n u)^{\theta_{1}}\right)^{3 \nu / 4}} \\
& \ll\left(\frac{c_{14} l}{u}\right)^{l} \frac{1}{(n u)^{3 \theta_{1} / 4}} .
\end{aligned}
$$

Inserting this into (2.5) and using the Stirling formula yield, for $1 \leq l \leq$ $c_{12} \log U$ and $U \leq u \leq 2 U$,

$$
\frac{T}{\left(b_{1} U\right)^{l}} \leq \frac{1}{l !}\left|h^{(l)}(u)\right|=\frac{\theta|\tau|}{2 \pi l u^{l}}+O\left(\left(\frac{c_{15}}{u}\right)^{l} \frac{|\tau|}{u^{\theta_{1} / 2}}\right) \leq \frac{T}{\left(b_{0} U\right)^{l}},
$$

where $b_{i}=b_{i}\left(\alpha, \alpha^{\prime}, \theta, \theta^{\prime}, \theta^{\prime \prime}, C\right)$ are constants satisfying $0<b_{0}<1<b_{1}$.

Under our assumptions on $M, T, U, U^{\prime}$, it is easy to see that the condition of Lemma 1 is satisfied with $\delta=\theta_{1}, D=T, P=U, Q=U^{\prime}-U, b_{0}=b_{0}$ 
and $b_{1}=b_{1}$. Thus Lemma 1 implies

$$
\sum_{U<r \leq U^{\prime}} e(h(r)) \ll U e^{-c_{16}(\log M)^{3} /(\log T)^{2}} .
$$

Inserting this estimate into (2.4), we obtain

$$
\begin{aligned}
S_{N}^{\prime}(\tau) & \ll M^{1-\delta}+M e^{-c_{16}(\log M)^{3} /(\log T)^{2}} \log M \\
& \ll M e^{-c_{17}(\log M)^{3} /(\log T)^{2}} .
\end{aligned}
$$

In view of $S_{N}^{\prime \prime}(\tau)$, it suffices to deal with the sum

$$
S_{N}^{\prime \prime}(\tau, j, Q):=\sum_{M^{1 / 2-\delta<k \leq N / Q}}\left|\sum_{\substack{Q<m \leq 2 Q \\ m \leq N / \max \{j, k\}}}\left(\frac{g_{0}(k m)}{g_{0}(j m)}\right)^{i \tau}\right|,
$$

where $M^{1 / 2-\delta} \leq Q \leq N / M^{1 / 2-\delta}$ and $M^{1 / 2-\delta}<j \leq N / Q$.

The contribution of the sum over $k$ such that $|j-k| \leq M^{1 / 2-2 \delta}$ is $\ll M^{1 / 2-2 \delta} Q \ll M^{1-\delta}$ and the sum over $k$ with $N / \max \{j, k\}<Q+Q^{1 / 2}$ is $\ll N Q^{-1 / 2} \ll M^{3 / 4+\delta / 2} \ll M^{1-\delta}$. Therefore we have, for $N / \max \{j, k\} \geq$ $Q+Q^{1 / 2}$

$$
S_{N}^{\prime \prime}(\tau, j, Q) \ll M^{1-\delta}+\sum_{\substack{M^{1 / 2-\delta}<k \leq N / Q \\|k-j|>M^{1 / 2-2 \delta}}}\left|\sum_{\substack{Q<m \leq 2 Q \\ m \leq N / \max \{j, k\}}}\left(\frac{g_{0}(k m)}{g_{0}(j m)}\right)^{i \tau}\right| .
$$

Define $h_{j, k}(u):=(\tau /(2 \pi)) \log \left(g_{0}(k u) / g_{0}(j u)\right)$. Similarly to $(2.5)$, we write

$$
\begin{aligned}
h_{j, k}(u)= & \frac{\tau}{2 \pi}\left\{\theta \log \frac{k}{j}+\frac{v(k u)}{\alpha(k u)^{\theta}}-\frac{v(j u)}{\alpha(j u)^{\theta}}\right. \\
& \left.-\sum_{\nu=2}^{\infty} \frac{(-1)^{\nu}}{\nu \alpha^{\nu}}\left[\left(\frac{v(k u)}{(k u)^{\theta}}\right)^{\nu}-\left(\frac{v(j u)}{(j u)^{\theta}}\right)^{\nu}\right]\right\} .
\end{aligned}
$$

Recall that $v(u)=\alpha^{\prime} u^{\theta^{\prime}}+t(u)$. By (2.6) with $t(n u)$ in place of $v(n u)$ and $\nu=1$, we have

$$
\begin{aligned}
& \frac{d^{l}}{d u^{l}}\left(\frac{v(k u)}{(k u)^{\theta}}-\frac{v(j u)}{(j u)^{\theta}}\right) \\
& \quad=\alpha^{\prime} \prod_{i=1}^{l}\left(1-\theta_{1}-i\right)\left(k^{-\theta_{1}}-j^{-\theta_{1}}\right) u^{-\theta_{1}-l}+\frac{d^{l}}{d u^{l}}\left(\frac{t(k u)}{(k u)^{\theta}}-\frac{t(j u)}{(j u)^{\theta}}\right) \\
& \quad=\alpha^{\prime} \prod_{i=1}^{l}\left(1-\theta_{1}-i\right)\left(k^{-\theta_{1}}-j^{-\theta_{1}}\right) u^{-\theta_{1}-l}+O\left(\frac{\left(c_{11} l\right)^{l} u^{-\theta_{2}-l}}{\min \{k, j\}^{\theta_{2}}}\right) .
\end{aligned}
$$


Similarly to (2.7), we can deduce that for $1 \leq l \leq c_{18} \log Q$,

$$
\begin{aligned}
\frac{h_{j, k}^{(l)}(u)}{l !}= & \frac{\tau}{2 \pi \alpha l !}\left\{\frac{d^{l}}{d u^{l}}\left(\frac{v(k u)}{(k u)^{\theta}}-\frac{v(j u)}{(j u)^{\theta}}\right)\right. \\
& \left.+O\left(\left(c_{19} l\right)^{l} \min \{k, j\}^{-3 \theta_{1} / 2} u^{-3 \theta_{1} / 2-l}\right)\right\} \\
= & \frac{\tau}{2 \pi \alpha l !}\left\{\alpha^{\prime} \prod_{i=1}^{l}\left(1-\theta_{1}-i\right)\left(k^{-\theta_{1}}-j^{-\theta_{1}}\right) u^{-\theta_{1}-l}\right. \\
& +O\left(\left(c_{19} l\right)^{l} \min \{k, j\}^{-3 \theta_{1} / 2} u^{-3 \theta_{1} / 2-l}\right. \\
& \left.\left.+\left(c_{11} l\right)^{l} \min \{k, j\}^{-\theta_{2}} u^{-\theta_{2}-l}\right)\right\} \\
= & \frac{\alpha^{\prime} \tau}{2 \pi \alpha}\left\{\prod_{i=1}^{l}\left(\frac{1-\theta_{1}}{i}-1\right)\left(k^{-\theta_{1}}-j^{-\theta_{1}}\right) u^{-\theta_{1}-l}\right. \\
& \left.+O\left(c_{20}^{l} \min \{k, j\}^{-\theta_{0}} u^{-\theta_{0}-l}\right)\right\} .
\end{aligned}
$$

Since $M^{1 / 2-\delta} \leq j, k \leq M^{1 / 2+\delta}$, we have, in view of $(2.2)$,

$$
\begin{aligned}
\left|k^{-\theta_{1}}-j^{-\theta_{1}}\right| & \geq M^{(1 / 2+\delta)\left(-\theta_{1}-1\right)}|k-j| \geq M^{(1 / 2+\delta)\left(-\theta_{1}-1\right)+1 / 2-2 \delta} \\
& =M^{-\left(3+\theta_{1}\right) \delta-\theta_{1} / 2} \geq M^{-(1 / 2-\delta) \theta_{0}} \geq \min \{k, j\}^{-\theta_{0}} .
\end{aligned}
$$

Thus for $1 \leq l \leq c_{18} \log Q$, we have

$$
\frac{\left|h_{j, k}^{(l)}(u)\right|}{l !}=\frac{\left|\alpha^{\prime} \tau\left(k^{-\theta_{1}}-j^{-\theta_{1}}\right)\right| u^{-\theta_{1}-l}}{2 \pi \alpha}\left\{\prod_{i=1}^{l}\left|\frac{1-\theta_{1}}{i}-1\right|+O\left(c_{20}^{l} u^{-\left(\theta_{0}-\theta_{1}\right)}\right)\right\} .
$$

Since $\theta_{0}>\theta_{1}$, there exists $b_{i}^{\prime}=b_{i}^{\prime}\left(\alpha, \alpha^{\prime}, \theta, \theta^{\prime}, \theta^{\prime \prime}, C\right)$ with $0<b_{0}^{\prime}<1<b_{1}^{\prime}$ such that we have, for $1 \leq l \leq c_{18} \log Q$ and $Q \leq u \leq 2 Q$,

$$
\frac{T\left|k^{-\theta_{1}}-j^{-\theta_{1}}\right| Q^{-\theta_{1}}}{\left(b_{1}^{\prime} Q\right)^{l}} \leq \frac{\left|h_{j, k}^{(l)}(u)\right|}{l !} \leq \frac{T\left|k^{-\theta_{1}}-j^{-\theta_{1}}\right| Q^{-\theta_{1}}}{\left(b_{0}^{\prime} Q\right)^{l}} .
$$

We then consider the following two cases according to the size of $T$. Let $\Theta:=(1+2 \delta) \theta_{1}+4 \delta$.

CASE 1: $M^{\Theta} \leq T \leq \exp \left\{\varrho_{3}(\log M)^{2}\right\}$. We appeal to Lemma 1. In view of $(2.10)$, we take

$$
D=T\left|k^{-\theta_{1}}-j^{-\theta_{1}}\right| Q^{-\theta_{1}} .
$$

Since $\max \{j, k, Q\} \leq M^{1 / 2+\delta}$ and $|j-k| \geq M^{1 / 2-2 \delta}$, we have

$$
\begin{aligned}
D & \geq M^{(1+2 \delta) \theta_{1}+4 \delta-(1 / 2+\delta)\left(1+\theta_{1}\right)+1 / 2-2 \delta} Q^{-\theta_{1}}=M^{\delta+\theta_{1} / 2+\theta_{1} \delta} Q^{-\theta_{1}} \\
& \geq Q^{\left(\delta+\theta_{1} / 2+\theta_{1} \delta\right) /(1 / 2+\delta)-\theta_{1}}=Q^{\delta /(1 / 2+\delta)} \geq Q^{\delta},
\end{aligned}
$$


and

$$
\begin{aligned}
\log D /\left(8 \delta_{0}^{2} \log Q\right)+1 & \leq \log T /\left(4 \delta_{0}^{2} \log Q\right) \\
& \leq \varrho_{3}(\log M)^{2} /\left(4 \delta_{0}^{2} \log Q\right) \leq c_{18} \log Q
\end{aligned}
$$

Lemma 1 yields

$$
\sum_{\substack{Q<m \leq 2 Q \\ m \leq N / \max \{j, k\}}}\left(\frac{g_{0}(k m)}{g_{0}(j m)}\right)^{i \tau} \ll Q \exp \left\{-c_{20} \frac{\log ^{3} M}{\log ^{2} T}\right\} .
$$

CAsE 2: $M^{\theta_{1}} \leq T \leq M^{\Theta}$. We apply van der Corput's classical result ([6], Theorem 2.9): if $h \in \mathcal{C}^{2}[Q, 2 Q]$ satisfies $h^{(l)}(u) \asymp H / Q^{l}$ for $l=1,2$ and $Q \leq u \leq 2 Q$, then

$$
\sup _{Q \leq Q_{1} \leq 2 Q}\left|\sum_{Q<n \leq Q_{1}} e(h(n))\right| \ll H^{1 / 2} Q^{1 / 2}+H^{-1} Q .
$$

The relation (2.10) shows that this result is applicable to $h=h_{j, k}$ with $H=T\left|k^{-\theta_{1}}-j^{-\theta_{1}}\right| Q^{-\theta_{1}}$. Since $\min \{j, k, Q\} \geq M^{1 / 2-\delta}$ and $\max \{j, k\} \leq$ $N / Q \leq 2 M / Q$, we have

$$
\left\{\begin{array}{l}
H \geq T \max \{j, k\}^{-\theta_{1}-1}|j-k| Q^{-\theta_{1}} \gg T Q|k-j| / M^{1+\theta_{1}} \\
H \leq 2 M^{(1+2 \delta) \theta_{1}+4 \delta-2 \theta_{1}(1 / 2-\delta)}=M^{4\left(1+\theta_{1}\right) \delta} \leq M^{1 / 3}
\end{array}\right.
$$

Thus we obtain

$$
\sum_{\substack{Q<m \leq 2 Q \\ m \leq N / \max \{j, k\}}}\left(\frac{g_{0}(k m)}{g_{0}(j m)}\right)^{i \tau} \ll M^{1 / 6} Q^{1 / 2}+\frac{M^{1+\theta_{1}}}{T|k-j|} .
$$

Now combining (2.11) and (2.12) with (2.9), we find

$$
\begin{aligned}
S_{N}^{\prime \prime}(\tau, j, Q) \ll & M^{1-\delta}+M e^{-c_{21}(\log M)^{3} /(\log T)^{2}} \\
& +M^{23 / 24}+T^{-1} M^{1+\theta_{1}} \log M \\
\ll & M e^{-c_{21}(\log M)^{3} /(\log T)^{2}}+T^{-1} M^{1+\theta_{1}} \log M .
\end{aligned}
$$

Finally inserting (2.8) and (2.13) into (2.3), we get

$$
\sum_{n \leq N} \Lambda(n) g_{0}(n)^{-i \tau} \ll M\left\{e^{-c_{22}(\log M)^{3} /(\log T)^{2}}+\left(M^{\theta_{1}} / T\right)^{1 / 2}(\log M)^{7 / 2}\right\},
$$

which implies the desired result. Our proof is complete.

The fourth lemma is an asymptotic formula on page 248 of [2]. Since the proof given there is quite sketchy, we present a detailed proof for convenience of the reader. The proof was provided by Balazard \& Tenenbaum and we reproduce it here with their permission. 
LEMMA 4. For any positive constants, given $\phi_{1}$ and $\phi_{2}$, there exists a positive constant $\varrho_{0}^{\prime}=\varrho_{0}^{\prime}\left(\phi_{1}, \phi_{2}\right)$ such that

$$
\sum_{n \leq z} \frac{\Lambda(n)}{n^{1+i \tau}}=-\frac{\zeta^{\prime}}{\zeta}(1+i \tau)+O\left(z^{-\varrho_{0}^{\prime} \beta(|\tau|+3)} \log z\right)
$$

for $e^{\phi_{1} /(\beta(|\tau|+3))} \leq z \leq(|\tau|+3)^{\phi_{2}}$.

Proof. Let $F(s):=-\zeta^{\prime}(s) / \zeta(s)$. By the Perron formula ([12], Theorem II.2.3), we can write

$$
U(z):=\sum_{n \leq z} \frac{\Lambda(n)}{n^{1+i \tau}} \log \left(\frac{z}{n}\right)=\frac{1}{2 \pi i} \int_{\xi-i \infty}^{\xi+i \infty} F(1+i \tau+w) \frac{z^{w}}{w^{2}} d w
$$

where $\xi:=1 / \log z$. According to Vinogradov-Korobov's well known bound, we have

$$
F(1+i \tau+w) \ll 1 /(\beta(|\tau+\operatorname{Im} w|+3)), \quad \operatorname{Re} w \geq-\varrho_{0}^{\prime \prime} \beta(|\tau+\operatorname{Im} w|+3),
$$

where $\varrho_{0}^{\prime \prime}>0$ is a constant. We truncate the integral in (2.14) to $|\operatorname{Im} w|=\sqrt{z}$ with an error

$$
\int_{\substack{\operatorname{Re} w=\xi \\|\operatorname{Im} w| \geq \sqrt{z}}} F(1+i \tau+w) \frac{z^{w}}{w^{2}} d w \ll \frac{\log z}{\sqrt{z}}
$$

where we have used (2.15) in the form

$$
\begin{aligned}
F(1+i \tau+w) & \ll \max \{1 /(\beta(|\tau|+3)), 1 /(\beta(|\operatorname{Im} w|+3))\} \\
& \ll \max \{\log z, \log (|\operatorname{Im} w|+3)\}
\end{aligned}
$$

for $\operatorname{Re} w=\xi$ and $e^{\phi_{1} /(\beta(|\tau|+3))} \leq z$. We move the segment of integration from $[\xi-i \sqrt{z}, \xi+i \sqrt{z}]$ to $\operatorname{Re} w=-2 \varrho_{0}^{\prime} \beta(|\tau|+3)$, where $\varrho_{0}^{\prime} \leq \varrho_{0}^{\prime \prime} / 10$ is a suitable positive constant depending on $\phi_{1}$ and $\phi_{2}$. The contribution of the vertical segment is

$$
\ll \frac{z^{-2 \varrho_{0}^{\prime} \beta(|\tau|+3)}}{\beta(|\tau|+3)} \int_{0}^{\sqrt{z}} \frac{d t}{t^{2}+\beta(|\tau|+3)^{2}} \ll \frac{z^{-2 \varrho_{0}^{\prime} \beta(|\tau|+3)}}{\beta(|\tau|+3)^{2}} \ll z^{-2 \varrho_{0}^{\prime} \beta(|\tau|+3)} \log ^{2} z
$$

and the contribution of the horizontal segments is

$$
\ll \frac{1}{z \beta(|\tau|+3)}\left(\frac{1}{\log z}+\beta(|\tau|+3)\right) \ll \frac{1}{z},
$$

where we have used $e^{\phi_{1} /(\beta(|\tau|+3))} \leq z \leq(|\tau|+3)^{\phi_{2}}$. Hence the residue theorem gives

$$
U(z)=F(1+i \tau) \log z+F^{\prime}(1+i \tau)+O\left(z^{-2 \varrho_{0}^{\prime} \beta(|\tau|+3)} \log ^{2} z\right) .
$$


From this, we deduce that for $\sqrt{z} \leq y \leq z^{2}$,

$$
\begin{aligned}
U(z+y)-U(z) & =\log \left(1+\frac{y}{z}\right) \sum_{n \leq z} \frac{\Lambda(n)}{n^{1+i \tau}}+\sum_{z<n \leq z+y} \frac{\Lambda(n)}{n^{1+i \tau}} \log \left(\frac{z+y}{n}\right) \\
& =\log (1+y / z) F(1+i \tau)+O\left(z^{-2 \varrho_{0}^{\prime} \beta(|\tau|+3)} \log ^{2} z\right) .
\end{aligned}
$$

Thus for $\sqrt{z} \leq y \leq z^{2}$ we obtain

$$
\sum_{n \leq z} \frac{\Lambda(n)}{n^{1+i \tau}}=F(1+i \tau)+O\left(\frac{z^{-2 \varrho_{0}^{\prime} \beta(|\tau|+3)} \log ^{2} z}{\log (1+y / z)}+\sum_{z<n \leq z+y} \frac{\Lambda(n)}{n}\right) .
$$

It remains to estimate the last sum in (2.16). Defining

$$
\psi(t):=\sum_{n \leq t} \Lambda(n)
$$

and using the Brun-Titchmarsh inequality ([12], Theorem I.4.9), we can deduce that for $\sqrt{z} \leq y \leq z^{2}$,

$$
\begin{aligned}
\sum_{z<n \leq z+y} \frac{\Lambda(n)}{n} & =\int_{z}^{z+y} \frac{d \psi(t)}{t}=\frac{\psi(z+y)}{z+y}-\frac{\psi(z)}{z}+\int_{z}^{z+y} \frac{\psi(t)}{t^{2}} d t \\
& =\frac{\psi(z+y)-\psi(z)}{z+y}-\frac{\psi(z) y}{(z+y) z}+\int_{z}^{z+y} \frac{\psi(t)}{t^{2}} d t \\
& \ll \frac{y}{z+y}+\log \left(1+\frac{y}{z}\right) \ll \log \left(1+\frac{y}{z}\right) .
\end{aligned}
$$

Take $y$ such that $\log (1+y / z)=z^{-\varrho_{0}^{\prime} \beta(|\tau|+3)} \log z$, i.e.

$$
y=z\left(z^{z^{-\varrho_{0}^{\prime} \beta(|\tau|+3)}}-1\right) \text {. }
$$

It is easy to see that $\sqrt{z} \leq z^{1-\varrho_{0}^{\prime} \beta(|\tau|+3)} \log z \leq y \leq z^{2}$. This completes the proof.

The last lemma is a variant of Hankel's formula ([12], Theorem II.5.2).

For $a \in \mathbb{R}$ and $r>0$, we use $\mathcal{H}(a, r)$ to denote the Hankel contour surrounding the point $s=a$ with radius $r$, which is defined as the path formed from the circle $|s-a|=r$ excluding the point $s=a-r$, together with the half-line $(-\infty, a-r]$ traced twice, with respective arguments $+\pi$ and $-\pi$. For each $X>|a-r|+1$, let $\mathcal{H}_{X}(a, r)$ be the part of the Hankel contour $\mathcal{H}(a, r)$ situated in the half-plane $\sigma>-X$.

Lemma 5. For $X>1, z \in \mathbb{C}$ and $k \in \mathbb{Z}^{+}$, we have

$$
\frac{1}{2 \pi i} \int_{\mathcal{H}_{X}(0, r)} s^{-z} e^{s}(\log s)^{k} d s=(-1)^{k} \frac{d^{k}}{d z^{k}}\left(\frac{1}{\Gamma(z)}\right)+E_{k, z}(X),
$$


where

$$
\left|E_{k, z}(X)\right| \leq \frac{e^{\pi|\operatorname{Im} z|}}{2 \pi} \int_{X}^{\infty} \sigma^{-\operatorname{Re} z} e^{-\sigma}(\log \sigma+\pi)^{k} d \sigma .
$$

Proof. According to the Hankel formula ([12], Theorem II.5.2), we have

$$
\frac{1}{2 \pi i} \int_{\mathcal{H}(0, r)} s^{-z} e^{s} d s=\frac{1}{\Gamma(z)} .
$$

Since the integral on the left-hand side is absolutely and uniformly convergent on any compact set in the $z$-plane, we can differentiate under the integral sign to obtain

$$
\frac{1}{2 \pi i} \int_{\mathcal{H}(0, r)} s^{-z} e^{s}(\log s)^{k} d s=(-1)^{k} \frac{d^{k}}{d z^{k}}\left(\frac{1}{\Gamma(z)}\right) .
$$

For $\sigma>1$ and $s=\sigma e^{ \pm i \pi}$, we have

$$
\left|s^{-z} e^{s}(\log s)^{k}\right| \leq \sigma^{-\operatorname{Re} z} e^{\pi|\operatorname{Im} z|-\sigma}(\log \sigma+\pi)^{k}
$$

This implies the desired estimate for $E_{k, z}(X)$.

3. Proof of Theorem 4. From the assumptions (1)-(3), we deduce that for $\sigma \geq 1 / \theta-\min \{\eta /(2 \theta), 1 / \theta-1 / \tilde{\theta}, 1 /(4 \theta)\}$,

$$
\begin{aligned}
\widetilde{\mathcal{F}}_{g}(s) & =\prod_{p}\left\{1+\frac{\kappa}{g(p)^{s}}+O\left(\frac{1}{p^{1+\eta / 2}}+\frac{1}{p^{\psi}}\right)\right\}\left\{1-\frac{\kappa}{\left(\alpha p^{\theta}\right)^{s}}+O\left(\frac{1}{p^{3 / 2}}\right)\right\} \\
& =\prod_{p}\left\{1+\kappa\left(\frac{1}{g(p)^{s}}-\frac{1}{\left(\alpha p^{\theta}\right)^{s}}\right)+O\left(\frac{1}{p^{1+\eta / 2}}+\frac{1}{p^{\psi}}+\frac{1}{p^{3 / 2}}\right)\right\},
\end{aligned}
$$

which implies, for $\sigma \geq 1 / \theta-\min \{\eta /(2 \theta), 1 / \theta-1 / \widetilde{\theta}, 1 /(4 \theta)\}$,

$$
\widetilde{\mathcal{F}}_{g}(s)=\exp \left\{\kappa \sum_{p}\left(g(p)^{-s}-\left(\alpha p^{\theta}\right)^{-s}\right)+O(1)\right\} \text {. }
$$

Noticing that $g(p)=\alpha p^{\theta}\left\{1+O\left(p^{-\theta_{1}}\right)\right\}$, we have

$$
g(p)^{-s}-\left(\alpha p^{\theta}\right)^{-s} \ll|s| p^{-\theta \sigma-\theta_{1}} \ll|s| p^{-1-\theta_{1} / 2} .
$$

This proves the assertion (i) provided $\varrho_{0} \leq \frac{1}{10} \min \{\eta /(2 \theta), 1 / \theta-1 / \widetilde{\theta}, 1 /(4 \theta)\}$.

In view of (3.1), in order to prove (ii), it suffices to show that for $\sigma \geq$ $1 / \theta-10 \varrho_{0} \beta(T)$,

$$
\left|\sum_{p}\left(g(p)^{-s}-\left(\alpha p^{\theta}\right)^{-s}\right)\right| \leq \alpha^{-\sigma}\left\{\frac{4}{3} \log _{2} T+\frac{2}{3} \log _{3} T+O(1)\right\} .
$$

We only need to consider the case of $g(p)=\alpha p^{\theta}+\alpha^{\prime} p^{\theta^{\prime}}+t(p)=g_{0}(p)$. 
Let $T_{0}:=e^{1 /\left(10 \theta \varrho_{0} \beta(T)\right)}$ and $T_{1}:=T^{\left(1+20 \theta \theta_{1}^{-1} \varrho_{0} \beta(T)\right) / \theta_{1}}$. Then we have

$$
\begin{aligned}
\sum_{p>T_{1}}\left|g_{0}(p)^{-s}-\left(\alpha p^{\theta}\right)^{-s}\right| & \ll|s| \sum_{p>T_{1}} p^{-\left(\theta \sigma+\theta_{1}\right)} \\
& \ll T \sum_{p>T_{1}} p^{-\left(1+\theta_{1}-10 \theta \varrho_{0} \beta(T)\right)} \ll 1
\end{aligned}
$$

provided $\varrho_{0} \leq \theta_{1} /(20 \theta \beta(3))$. Noticing that

$$
\begin{aligned}
\left|g_{0}(p)^{-s}-\left(\alpha p^{\theta}\right)^{-s}\right| & \leq 2\left(\alpha p^{\theta}\left\{1+O\left(p^{-\theta_{1}}\right)\right\}\right)^{-\sigma} \\
& \leq 2 \alpha^{-\sigma} p^{-1+10 \theta \varrho_{0} \beta(T)}\left\{1+O\left(p^{-\theta_{1}}\right)\right\},
\end{aligned}
$$

we deduce

$$
\begin{aligned}
\left|\sum_{p \leq T_{0}}\left(g_{0}(p)^{-s}-\left(\alpha p^{\theta}\right)^{-s}\right)\right| & \leq 2 \alpha^{-\sigma}\left\{\sum_{p \leq T_{0}} \frac{1+O(\beta(T) \log p)}{p}+O(1)\right\} \\
& \leq \alpha^{-\sigma}\left\{\frac{4}{3} \log _{2} T+\frac{2}{3} \log _{3} T+O(1)\right\} .
\end{aligned}
$$

By using Lemma 4, we have

$$
\begin{aligned}
\sum_{T_{0}<p \leq T_{1}} \frac{1}{\left(\alpha p^{\theta}\right)^{s}} & =\alpha^{-s} \sum_{T_{0}<n \leq T_{1}} \frac{\Lambda(n)}{n^{\theta s} \log n}+O(1) \\
& =\alpha^{-s} \int_{T_{0}}^{T_{1}} \frac{z^{1-\theta \sigma}}{\log z} d\left(\sum_{n \leq z} \frac{\Lambda(n)}{n^{1+i \theta \tau}}\right)+O(1) \\
& =\alpha^{-s} \int_{T_{0}}^{T_{1}} \frac{z^{1-\theta \sigma}}{\log z} d O\left(z^{-2 c_{23} \beta(T)} \log z\right)+O(1) \\
& \ll \alpha^{-\sigma}\left\{1+\left(|1-\theta \sigma|+\frac{1}{\log T_{0}}\right) \int_{T_{0}}^{T_{1}} \frac{d z}{z^{1+c_{23} \beta(T)}}\right\} \ll 1,
\end{aligned}
$$

provided $\varrho_{0} \leq c_{23} /(10 \theta)$.

Our remaining task is to show

$$
\sum_{T_{0}<p \leq T_{1}} g_{0}(p)^{-s} \ll 1 .
$$

We divide this sum into two parts according as $T_{0}<p \leq T^{\prime}$ or $T^{\prime}<p \leq T_{1}$, where $T^{\prime}:=T^{\left(1-\varrho_{1}^{2} \beta(T)\right) / \theta_{1}}$ and $\varrho_{1}$ is the constant determined by Lemma 2 . We use $W_{1}$ and $W_{2}$ to denote the corresponding contribution and apply Lemmas 3 and 2 to treat them. 
Let $M_{j}:=\min \left\{2^{j} T^{\prime}, T_{1}\right\}$ and $J \in \mathbb{N}$ such that $2^{J} T^{\prime} \leq T_{1}<2^{J+1} T^{\prime}$. We have

$$
\begin{aligned}
W_{2} & =\sum_{j=0}^{J} \int_{M_{j}}^{M_{j+1}} \frac{1}{g_{0}(z)^{\sigma} \log z} d\left(\sum_{M_{j}<n \leq z} \Lambda(n) g_{0}(n)^{-i \tau}\right)+O(1) \\
& \ll \sum_{j=0}^{J} M_{j}^{-\theta \sigma} S_{M_{j}}(\tau)+1 .
\end{aligned}
$$

The choice of $T^{\prime}$ and the fact that $T^{1 /\left(2 \theta_{1}\right)} \leq T^{\prime} \leq M_{j} \leq T_{1} \leq T^{2 / \theta_{1}}$ guarantee that Lemma 2 is applicable. Thus we deduce that, provided $\varrho_{0} \leq$ $\varrho_{1} /(20 \theta)$,

$$
\begin{aligned}
W_{2} & \ll \sum_{j=0}^{J} M_{j}^{10 \theta \varrho_{0} \beta(T)}\left(M_{j}^{-\varrho_{1} \beta(T)}+T^{-1}+\mathcal{L}\left(M_{j}\right)^{-\varrho_{1}}\right)+1 \\
& \ll \sum_{j=0}^{J}\left(2^{j} T^{\prime}\right)^{-\varrho_{1} \beta(T) / 2}+1 \ll 1 .
\end{aligned}
$$

Let $N_{k}:=\min \left\{2^{k} T_{0}, T^{\prime}\right\}$ and $K \in \mathbb{N}$ such that $2^{K} T_{0} \leq T^{\prime}<2^{K+1} T_{0}$. As before we have

$$
W_{1} \ll \sum_{k=0}^{K} N_{k}^{-\theta \sigma} S_{N_{k}}(\tau)+1 .
$$

Assume that $\varrho_{0} \leq \varrho_{3}^{2 / 3} /(10 \theta)$, where $\varrho_{3}$ is given in Lemma 3. Since

$$
\begin{aligned}
N_{k}^{\theta_{1}} & \leq T^{\prime \theta_{1}} \leq T \\
& \leq \exp \left\{\left(10 \theta \varrho_{0} \log T_{0}\right)^{3 / 2}\right\} \\
& \leq \exp \left\{\varrho_{3}\left(\log T_{0}\right)^{2}\right\} \leq \exp \left\{\varrho_{3}\left(\log N_{k}\right)^{2}\right\},
\end{aligned}
$$

Lemma 3 is applicable. Thus

$$
\begin{aligned}
W_{1} & \ll \sum_{k=0}^{K} N_{k}^{10 \theta \varrho_{0} \beta(T)}\left(e^{-\varrho_{2}\left(\log N_{k}\right)^{3} /(\log T)^{2}}+\left(N_{k}^{\theta_{1}} / T\right)^{1 / 2}\left(\log N_{k}\right)^{4}\right) \\
& \ll(\log T)^{4} \sum_{k=0}^{K} 2^{10 \theta \varrho_{0} \beta(T) k}\left(e^{-\varrho_{2}\left(\log N_{k}\right)^{3} /(\log T)^{2}}+T^{-\varrho_{1}^{2} \beta(T) / 2}\right) .
\end{aligned}
$$

Noticing that

$$
\left(\log N_{k}\right)^{3} /(\log T)^{2} \geq k^{3} /\left(4(\log T)^{2}\right)+\log _{2} T /\left(10 \theta \varrho_{0}\right)^{3}
$$

for $0 \leq k \leq K$, we deduce 


$$
\begin{aligned}
W_{1} \ll & (\log T)^{5}\left\{(\log T)^{-\varrho_{2} /\left(10 \theta \varrho_{0}\right)^{3}} \sup _{k \geq 0} 2^{10 \theta \varrho_{0} \beta(T) k} e^{-\varrho_{2} k^{3} /\left(4(\log T)^{2}\right)}\right. \\
& \left.+T^{\left(10 \theta \varrho_{0} / \theta_{1}-\varrho_{1}^{2} / 2\right) \beta(T)}\right\} \\
& \ll(\log T)^{5-\varrho_{2} /\left(10 \theta \varrho_{0}\right)^{3}}+T^{\left(10 \theta \varrho_{0} / \theta_{1}-\varrho_{1}^{2} / 2\right) \beta(T)}(\log T)^{5} \ll 1,
\end{aligned}
$$

provided $\varrho_{0} \leq \min \left\{\left(\varrho_{2} / 10\right)^{1 / 3} /(10 \theta), \theta_{1} \varrho_{1}^{2} /(40 \theta)\right\}$. This completes the proof.

4. Proof of Theorem 1. For simplicity, we introduce the notations:

$$
\begin{gathered}
z_{j}:=\kappa / \alpha^{1 / \theta}-j, \quad M(x):=x^{1 / \theta}(\log x)^{\kappa / \alpha^{1 / \theta}-1}, \\
\frac{1}{\Gamma_{i}(a)}:=\left[\frac{d^{i}}{d z^{i}}\left(\frac{1}{\Gamma(z)}\right)\right]_{z=a} .
\end{gathered}
$$

Let $\xi=1 / \theta+1 / \log x$. By Theorem II.2.3 in [12], we can write

$$
\int_{0}^{x} F_{g}(t) d t=\frac{1}{2 \pi i} \int_{\xi-i \infty}^{\xi+i \infty} \mathcal{F}_{g}(s) \frac{x^{s+1}}{s(s+1)} d s .
$$

Let $\varrho_{4}<\varrho_{0}$ be a positive number small enough that

$$
\left\{\begin{array}{l}
|(\theta s-1) \log (\theta s-1)| \leq \theta \varrho_{0} / 2 \\
|\log (\theta s-1)| \geq 1
\end{array} \quad\left(|s-1 / \theta| \leq 10 \varrho_{4}\right) .\right.
$$

Let $U>1$ be a parameter to be chosen later. The residue theorem allows us to deform the segment of integration $[\xi-i U, \xi+i U]$ into the following path symmetrically with respect to the real axis. Its upper part is made up of: the upper portion (above the real axis) of the truncated Hankel contour $\mathcal{H}_{1}=\mathcal{H}_{4 \varrho_{4} \beta(3)-1 / \theta}\left(1 / \theta, \varrho_{4} / \log x\right)$, the curve $\sigma=1 / \theta-4 \varrho_{4} \beta(\tau+3)$ for $0 \leq \tau \leq U$; and the horizontal segment $\left[1 / \theta-4 \varrho_{4} \beta(U+3)+i U, \xi+i U\right]$.

From Theorem 4(ii) and the classic bound for $\zeta(s)([12]$, Note of Chapter II.3), we have

$$
\mathcal{F}_{g}(s) \ll(\log (|\tau|+3))^{c_{24}} \quad\left(\sigma>1 / \theta-10 \varrho_{0} \beta(|\tau|+3)\right) .
$$

Using this estimate, we easily see that the contribution from the vertical half-lines $[\xi \pm i U, \xi \pm i \infty]$ and from the horizontal segments

$$
\left[1 / \theta-4 \varrho_{4} \beta(U+3) \pm i U, \xi \pm i U\right]
$$

is $\ll x^{1+1 / \theta} / \sqrt{U}$.

Finally the integral over the arcs $\sigma=1 / \theta-4 \varrho_{4} \beta(|\tau|+3)(0 \leq|\tau| \leq U)$ is

$$
\ll x^{1+1 / \theta-4 \varrho_{4} \beta(U+3)} \int_{0}^{U} \frac{(\log (\tau+3))^{c_{24}}}{(\tau+1)^{2}} d \tau \ll x^{1+1 / \theta-4 \varrho_{4} \beta(U+3)} .
$$


Inserting these estimates into (4.1) and taking $U=\mathcal{L}(x)^{c_{25}}$, we can obtain

$$
\int_{0}^{x} F_{g}(t) d t=\Psi(x)+O\left(x^{1+1 / \theta} / \mathcal{L}(x)^{c_{26}}\right),
$$

where

$$
\Psi(x)=\frac{1}{2 \pi i} \int_{\mathcal{H}_{1}} \mathcal{F}_{g}(s) \frac{x^{s+1}}{s(s+1)} d s .
$$

Next we need to study the function $\Psi(x)$. Clearly it is an infinitely differentiable function of $x$ on $\mathbb{R}^{+}$, and we have

$$
\Psi^{\prime}(x)=\frac{1}{2 \pi i} \int_{\mathcal{H}_{1}} \mathcal{F}_{g}(s) \frac{x^{s}}{s} d s, \quad \Psi^{\prime \prime}(x)=\frac{1}{2 \pi i} \int_{\mathcal{H}_{1}} \mathcal{F}_{g}(s) x^{s-1} d s .
$$

Now for $s \notin(-\infty, 1 / \theta]$, we can write

$$
\begin{aligned}
(\theta s-1)^{-\kappa / \alpha^{s}+z_{0}} & =\exp \left\{z_{0}\left(1-\alpha^{-(\theta s-1) / \theta}\right) \log (\theta s-1)\right\} \\
& =\sum_{m=0}^{\infty} \frac{\left(z_{0}(\theta s-1) \log (\theta s-1)\right)^{m}}{m !}\left(\frac{1-\alpha^{-(\theta s-1) / \theta}}{\theta s-1}\right)^{m} .
\end{aligned}
$$

Note that for $m \geq 0$ we have

$$
\left(\frac{1-\alpha^{-(\theta s-1) / \theta}}{\theta s-1}\right)^{m}=\left(\frac{\log \alpha}{\theta}\right)^{m} \sum_{n=0}^{\infty} b_{m, n}\left(-\frac{\log \alpha}{\theta}\right)^{n}(\theta s-1)^{n},
$$

where $b_{m, n}$ is defined as in Section 1. Obviously

$$
b_{m, n} \leq \sum_{n_{1}+\ldots+n_{m}=n} \frac{1}{n_{1} ! \ldots n_{m} !}=\frac{m^{n}}{n !} .
$$

It follows that

$$
\begin{aligned}
(\theta s-1)^{-\kappa / \alpha^{s}+z_{0}} & \\
& =\sum_{m=0}^{\infty} \frac{\left(z_{0} \log (\theta s-1)\right)^{m}}{m !} \sum_{n=0}^{\infty}(-1)^{n} b_{m, n}\left(\frac{\log \alpha}{\theta}\right)^{m+n}(\theta s-1)^{m+n} \\
& =\sum_{m=0}^{\infty}\left\{\left(\frac{\log \alpha}{\theta}\right)^{m} \sum_{k=0}^{m} \frac{(-1)^{m-k} b_{k, m-k}}{k !}\left(z_{0} \log (\theta s-1)\right)^{k}\right\}(\theta s-1)^{m} .
\end{aligned}
$$

Noticing that $s^{-1} \mathcal{F}_{g}(s)=s^{-1} \widetilde{\mathcal{F}}_{g}(s)\{\zeta(\theta s)(\theta s-1)\}^{\kappa / \alpha^{s}}(\theta s-1)^{-\kappa / \alpha^{s}}$ and 
using (1.3) and (1.4), we deduce that, for $s \notin(-\infty, 1 / \theta]$ and $|s-1 / \theta|<10 \varrho_{0}$,

$$
\begin{aligned}
& \text { (4.6) } \quad \frac{\mathcal{F}_{g}(s)}{s} \\
& =\sum_{j=0}^{\infty}\left\{\sum_{m=0}^{j} \frac{a_{j-m}(\log \alpha)^{m}}{\theta^{j}} \sum_{k=0}^{m} \frac{(-1)^{m-k} b_{k, m-k}}{k !}\left(z_{0} \log (\theta s-1)\right)^{k}\right\}(\theta s-1)^{-z_{j}} \\
& =\sum_{j=0}^{\infty} \sum_{k=0}^{j} \frac{e_{j, k}}{\theta^{j}}(\theta s-1)^{-z_{j}}(\log (\theta s-1))^{k}
\end{aligned}
$$

where

$$
\begin{aligned}
e_{j, k} & :=\frac{z_{0}^{k}}{k !} \sum_{m=k}^{j}(-1)^{m-k}(\log \alpha)^{m} a_{j-m} b_{k, m-k} \\
& \ll \frac{\left|z_{0}\right|^{k}}{k !} \sum_{m=k}^{j} \frac{|\log \alpha|^{m}}{\varrho_{0}^{j-m}} \cdot \frac{k^{m-k}}{(m-k) !} \\
& \ll \frac{\left(\varrho_{0}\left|z_{0} \log \alpha\right|\right)^{k}}{\varrho_{0}^{j} k !} \sum_{n=0}^{j-k} \frac{\left(\varrho_{0} k|\log \alpha|\right)^{n}}{n !} \\
& \ll \varrho_{0}^{-j} \frac{\left(\varrho_{0}\left|z_{0} \log \alpha\right| e^{\varrho_{0}|\log \alpha|}\right)^{k}}{k !} \\
& \ll \varrho_{0}^{-j} \frac{\lambda^{k}}{k !} .
\end{aligned}
$$

For any integer $J \geq 0$, we split the double sum in (4.6) into two parts to obtain

$$
\Psi^{\prime}(x)=M_{J}(x)+\Xi_{J}(x),
$$

where

$$
\begin{aligned}
& M_{J}(x):=\sum_{j=0}^{J} \sum_{k=0}^{j} \frac{e_{j, k}}{\theta^{j}} \cdot \frac{1}{2 \pi i} \int_{\mathcal{H}_{1}} x^{s}(\theta s-1)^{-z_{j}}(\log (\theta s-1))^{k} d s, \\
& \Xi_{J}(x):=\frac{1}{2 \pi i} \int_{\mathcal{H}_{1}} x^{s} \sum_{j=J+1}^{\infty} \sum_{k=0}^{j} \frac{e_{j, k}}{\theta^{j}}(\theta s-1)^{-z_{j}}(\log (\theta s-1))^{k} d s .
\end{aligned}
$$

Observing that $\mathcal{H}_{1}$ is contained in the disc $|s-1 / \theta| \leq 10 \varrho_{4}$, the inequalities (4.2) and (4.7) allow us to deduce that the double sum in $\Xi_{J}(x)$ is, for $s \in \mathcal{H}_{1}$,

$$
\ll|\theta s-1|^{-\operatorname{Re} z_{0}} \sum_{j>J} \sum_{k \leq j} \frac{|\lambda \log (\theta s-1)|^{k}}{k !} \cdot \frac{|\theta s-1|^{j}}{\left(\theta \varrho_{0}\right)^{j}}
$$




$$
\begin{aligned}
& \ll|\theta s-1|^{-\operatorname{Re} z_{0}} \sum_{j>J}\left(1+|\lambda \log (\theta s-1)|^{j}\right) \frac{|\theta s-1|^{j}}{\left(\theta \varrho_{0}\right)^{j}} \\
& \ll|\theta s-1|^{-\operatorname{Re} z_{0}}\left(\frac{|\theta s-1|^{J+1}}{\left(\theta \varrho_{0}\right)^{J+1}}+\frac{|\lambda(\theta s-1) \log (\theta s-1)|^{J+1}}{\left(\theta \varrho_{0}\right)^{J+1}}\right) .
\end{aligned}
$$

Noticing that $|\log (\theta s-1)| \leq \log _{2} x+c_{27}\left(s \in \mathcal{H}_{1}\right)$, we find that for $s \in \mathcal{H}_{1}$,

$$
\begin{aligned}
\sum_{j=J+1}^{\infty} \sum_{k=0}^{j} \frac{e_{j, k}}{\theta^{j}}(\theta s-1)^{-z_{j}} & (\log (\theta s-1))^{k} \\
& \ll|\theta s-1|^{-\operatorname{Re} z_{0}}\left(\frac{\lambda \log _{2} x+c_{28}}{\theta \varrho_{0}}|\theta s-1|\right)^{J+1} .
\end{aligned}
$$

From this we deduce that

$$
\Xi_{J}(x) \ll\left(\lambda \log _{2} x+c_{28}\right)^{J+1} \Xi_{J}^{*}(x),
$$

where

$$
\begin{aligned}
\Xi_{J}^{*}(x) & :=\frac{1}{\left(\theta \varrho_{0}\right)^{J+1}} \int_{1 / \theta-4 \varrho_{4} \beta(3)}^{1 / \theta-r} x^{\sigma}|1-\theta \sigma|^{J+1-\operatorname{Re} z_{0}} d \sigma+\frac{x^{1 / \theta+r}}{r^{\operatorname{Re} z_{0}-J-2}} \\
& \ll \frac{|M(x)|}{\left(\theta \varrho_{0} \log x\right)^{J+1}}\left\{\int_{\varrho_{4}}^{4 \varrho_{4} \beta(3) \log x} t^{J+1-\operatorname{Re} z_{0}} e^{-t} d t+1\right\} \\
& \ll \frac{|M(x)|}{\left(\theta \varrho_{0} \log x\right)^{J+1}}\left\{\Gamma\left(J+2-\operatorname{Re} z_{0}\right)+1\right\} \\
& \ll|M(x)|\left(\frac{c_{29} J+1}{\log x}\right)^{J+1} .
\end{aligned}
$$

Thus

$$
\Xi_{J}(x) \ll|M(x)|\left(\left(c_{29} J+1\right) \frac{\lambda \log _{2} x+c_{28}}{\log x}\right)^{J+1} .
$$

Using the change of variable $w=(s-1 / \theta) \log x$ and Lemma 5 , we have, with the notation $\mathcal{H}_{0}:=\mathcal{H}_{X}\left(0, \varrho_{4}\right)$ and $X:=4 \varrho_{4} \beta(3) \log x$,

$$
\begin{aligned}
M_{J}(x)= & M(x) \sum_{j=0}^{J} \frac{\theta^{-z_{0}}}{(\log x)^{j}} \sum_{k=0}^{j} \frac{e_{j, k}}{2 \pi i} \int_{\mathcal{H}_{0}} \frac{e^{w}\{\log w+\log (\theta / \log x)\}^{k}}{w^{z_{j}}} d w \\
= & M(x) \sum_{j=0}^{J} \frac{\theta^{-z_{0}}}{(\log x)^{j}} \sum_{k=0}^{j} e_{j, k} \sum_{i=0}^{k}\left(\begin{array}{c}
k \\
i
\end{array}\right)\left(\log \frac{\theta}{\log x}\right)^{k-i} \\
& \times\left\{\frac{(-1)^{i}}{\Gamma_{i}\left(z_{j}\right)}+E_{k, z_{j}}(X)\right\} .
\end{aligned}
$$


The contribution of $(-1)^{i} / \Gamma_{i}\left(z_{j}\right)$ to $M_{J}(x)$ is

$$
\begin{aligned}
M(x) \sum_{j=0}^{J} \frac{\theta^{-z_{0}}}{(\log x)^{j}} \sum_{k=0}^{j} e_{j, k} \sum_{i=0}^{k}\left(\begin{array}{l}
k \\
i
\end{array}\right)\left(\log \frac{\theta}{\log x}\right)^{k-i} & \frac{(-1)^{i}}{\Gamma_{i}\left(z_{j}\right)} \\
& =M(x) \sum_{j=0}^{J} \frac{P_{j}\left(\log _{2} x\right)}{(\log x)^{j}},
\end{aligned}
$$

where

$$
\begin{aligned}
P_{j}(t) & :=\frac{1}{\theta^{z_{0}}} \sum_{k=0}^{j} e_{j, k} \sum_{i=0}^{k}(-1)^{i}\left(\begin{array}{c}
k \\
i
\end{array}\right) \frac{(\log \theta-t)^{k-i}}{\Gamma_{i}\left(z_{j}\right)} \\
& =\frac{1}{\theta^{z_{0}}} \sum_{k=0}^{j} e_{j, k} \sum_{i=0}^{k}(-1)^{i}\left(\begin{array}{c}
k \\
i
\end{array}\right) \sum_{l=0}^{k-i}\left(\begin{array}{c}
k-i \\
l
\end{array}\right) \frac{(\log \theta)^{k-i-l}}{\Gamma_{i}\left(z_{j}\right)}(-t)^{l} \\
& =\frac{1}{\theta^{z_{0}}} \sum_{k=0}^{j} e_{j, k} \sum_{l=0}^{k} \sum_{i=0}^{k-l}(-1)^{i}\left(\begin{array}{c}
k \\
i
\end{array}\right)\left(\begin{array}{c}
k-i \\
l
\end{array}\right) \frac{(\log \theta)^{k-i-l}}{\Gamma_{i}\left(z_{j}\right)}(-t)^{l} \\
& =\frac{1}{\theta^{z_{0}}} \sum_{l=0}^{j} \sum_{k=l}^{j} e_{j, k} \sum_{i=0}^{k-l}(-1)^{i}\left(\begin{array}{c}
k \\
i
\end{array}\right)\left(\begin{array}{c}
k-i \\
l
\end{array}\right) \frac{(\log \theta)^{k-i-l}}{\Gamma_{i}\left(z_{j}\right)}(-t)^{l} \\
& =\sum_{l=0}^{j} \lambda_{j, l} t^{l}
\end{aligned}
$$

and from (4.7),

$$
\begin{aligned}
\lambda_{j, l}:= & \frac{(-1)^{l}}{\theta^{z_{0}}} \sum_{k=l}^{j} e_{j, k} \sum_{i=0}^{k-l}(-1)^{i}\left(\begin{array}{c}
k \\
i
\end{array}\right)\left(\begin{array}{c}
k-i \\
l
\end{array}\right) \frac{(\log \theta)^{k-l-i}}{\Gamma_{i}\left(z_{j}\right)} \\
= & \frac{\theta^{-z_{0}}}{l !} \sum_{k=l}^{j}\left(\kappa \alpha^{-1 / \theta}\right)^{k} \\
& \times \sum_{m=k}^{j}(-\log \alpha)^{m} a_{j-m} b_{k, m-k} \sum_{i=0}^{k-l} \frac{(-\log \theta)^{k-l-i}}{(k-l-i) ! i ! \Gamma_{i}\left(z_{j}\right)} \\
= & \frac{\theta^{-\kappa / \alpha^{1 / \theta}}}{l !} \sum_{m=l}^{j} \sum_{k=l}^{m} \sum_{i=0}^{k-l} \lambda_{m, k, i}^{*},
\end{aligned}
$$

where

$$
\lambda_{m, k, i}^{*}:=\frac{(-\log \alpha)^{m}\left(\kappa \alpha^{-1 / \theta}\right)^{k}(-\log \theta)^{k-l-i} a_{j-m} b_{k, m-k}}{(k-l-i) ! i ! \Gamma_{i}\left(\kappa / \alpha^{1 / \theta}-j\right)}
$$


The contribution of $E_{k, z_{j}}(X)$ to $M_{J}(x)$ is, via (2.17),

$$
\begin{aligned}
& \ll|M(x)| \sum_{j=0}^{J} \sum_{k=0}^{j} \frac{\left|e_{j, k}\right|}{(\log x)^{j}} \sum_{i=0}^{k}\left(\begin{array}{c}
k \\
i
\end{array}\right)\left(\log \frac{\theta}{\log x}\right)^{k-i} \\
& \quad \times \int_{X}^{\infty} \sigma^{\left|z_{j}\right|}(\log \sigma+\pi)^{i} e^{-\sigma} d \sigma \\
& \ll|M(x)| \sum_{j=0}^{J} \frac{1}{\left(\varrho_{0} \log x\right)^{j}} \sum_{k=0}^{j} \frac{\lambda^{k}}{k !} \int_{X}^{\infty}\left(2 \log _{2} x+2 \log \sigma\right)^{k} \sigma^{\left|z_{j}\right|} e^{-\sigma} d \sigma \\
& \ll|M(x)| \sum_{j=0}^{J} \frac{1}{\left(\varrho_{0} \log x\right)^{j}} \int_{X}^{\infty} e^{2 \lambda \log _{2} x+2 \lambda \log \sigma} \sigma^{\left|z_{j}\right|} e^{-\sigma} d \sigma \\
& \ll|M(x)| \sum_{j=0}^{J} \frac{(\log x)^{2 \lambda}}{\left(\varrho_{0} \log x\right)^{j}} \int_{X}^{\infty} \sigma^{\left|z_{j}\right|+2 \lambda} e^{-\sigma} d \sigma .
\end{aligned}
$$

Since

$$
\begin{aligned}
\int_{X}^{\infty} \sigma^{\left|z_{j}\right|+2 \lambda} e^{-\sigma} d \sigma & \ll e^{-X / 2} \int_{X}^{\infty} \sigma^{\left|z_{j}\right|+2 \lambda} e^{-\sigma / 2} d \sigma \\
& \ll e^{-X / 2} 2^{j} \int_{X / 2}^{\infty} \sigma^{\left|z_{j}\right|+2 \lambda} e^{-\sigma} d \sigma \\
& \ll e^{-X / 2} 2^{j} \Gamma\left(j+\left[\left|z_{0}\right|+2 \lambda\right]+2\right) \\
& \ll e^{-X / 2}\left(\left|2 z_{0}\right|+4 \lambda+4\right)^{j}(j+1) !
\end{aligned}
$$

the contribution of $E_{k, z_{j}}(X)$ to $M_{J}(x)$ is

$$
\begin{aligned}
& \ll|M(x)| e^{-X / 4} \sum_{j=0}^{J}\left(\frac{\left|2 z_{0}\right|+4 \lambda+4}{\varrho_{0} \log x}\right)^{j}(j+1) ! \\
& \ll|M(x)| e^{-X / 4} \sum_{j=0}^{J}\left(\frac{8}{X}\right)^{j}(j+1) ! \\
& \ll|M(x)| e^{-X / 4}\left(\frac{8}{X}\right)^{J} \sum_{j=0}^{J}\left(\frac{X}{8}\right)^{J-j} \frac{(J+1) !}{(J-j) !} \\
& \ll|M(x)| e^{-X / 8}\left(\frac{8}{X}\right)^{J}(J+1) ! \ll|M(x)|\left(\frac{c_{29} J+1}{\log x}\right)^{J+1}
\end{aligned}
$$

provided $\varrho_{4} \leq \varrho_{0} /\left(\beta(3)\left(\left|z_{0}\right|+2 \lambda+2\right)\right)$. Combining these estimates yields

$$
M_{J}(x)=M(x)\left\{\sum_{j=0}^{J} \frac{P_{j}\left(\log _{2} x\right)}{(\log x)^{j}}+O\left(\left(\frac{c_{29} J+1}{\log x}\right)^{J+1}\right)\right\} .
$$


Inserting (4.9) and (4.10) into (4.8), we obtain

$$
\begin{aligned}
& \Psi^{\prime}(x) \\
& =M(x)\left\{\sum_{j=0}^{J} \frac{P_{j}\left(\log _{2} x\right)}{(\log x)^{j}}+O\left(\left(\left(c_{29} J+1\right) \frac{\lambda \log _{2} x+c_{28}}{\log x}\right)^{J+1}\right)\right\} .
\end{aligned}
$$

Obviously, we have

$$
\Psi^{\prime \prime}(x)=\frac{1}{2 \pi i} \int_{\mathcal{H}_{1}} \mathcal{F}_{g}(s) x^{s-1} d s \ll x^{1 / \theta-1}(\log x)^{|\kappa| / \alpha^{1 / \theta}} .
$$

Assume that $f(n) \geq 0$ for all integers $n$. Then, with (4.4), we have

$$
\begin{aligned}
h F_{g}(x) \leq & \int_{x}^{x+h} F_{g}(t) d t \\
= & \Psi(x+h)-\Psi(x)+O\left(x^{1+1 / \theta} \mathcal{L}(x)^{-c_{26}}\right) \\
= & h \Psi^{\prime}(x)+h^{2} \int_{0}^{1}(1-t) \Psi^{\prime \prime}(x+t h) d t \\
& +O\left(x^{1+1 / \theta} \mathcal{L}(x)^{-c_{26}}\right) \\
= & h \Psi^{\prime}(x)+O\left(h^{2} x^{1 / \theta-1}(\log x)^{|\kappa| / \alpha^{1 / \theta}}+x^{1+1 / \theta} \mathcal{L}(x)^{-c_{26}}\right) .
\end{aligned}
$$

Taking $h=x \mathcal{L}(x)^{-c_{30}}$, we obtain

$$
F_{g}(x) \leq \Psi^{\prime}(x)+O\left(x^{1 / \theta} \mathcal{L}(x)^{-c_{3}}\right) .
$$

Similarly, we can prove

$$
F_{g}(x) \geq \frac{1}{h} \int_{x-h}^{x} F_{g}(t) d t \geq \Psi^{\prime}(x)+O\left(x^{1 / \theta} \mathcal{L}(x)^{-c_{3}}\right) .
$$

This proves the desired asymptotic formula in the case of $f(n) \geq 0$.

For the general case, write $\left|F_{g}\right|(x)=\sum_{g(n) \leq x}|f(n)|$. We first check that the conditions in Theorem 1 are satisfied by $\left|F_{g}\right|$. Since

$$
\left|F_{g}(x)-\frac{1}{h} \int_{x}^{x+h} F_{g}(t) d t\right| \leq \frac{1}{h} \int_{x}^{x+h}\left|F_{g}\right|(t) d t-\left|F_{g}\right|(x),
$$

the desired asymptotic formula then follows from (4.11) and (4.12).

Finally we prove (1.9). Noticing that for $0 \leq \alpha \leq \beta$,

$$
\begin{aligned}
\int_{1}^{\infty} e^{-\sigma} \sigma^{\beta}(\log \sigma)^{\alpha} d \sigma & \ll(\log (\alpha+2))^{\alpha} \int_{1}^{\infty} e^{-\sigma / 2} \sigma^{\beta} d \sigma \\
& \ll(\log (\alpha+2))^{\alpha}\left(c_{31} \beta+1\right)^{\beta}
\end{aligned}
$$


we deduce

$$
\begin{aligned}
\frac{1}{\left|\Gamma_{n}\left(z_{j}\right)\right|} & =\left|\frac{1}{2 \pi i} \int_{\mathcal{H}(0,1)} s^{-z_{j}} e^{s}(\log s)^{n} d s\right| \\
& \ll \int_{1}^{\infty} \sigma^{\left|z_{0}\right|+j} e^{-\sigma}(\log \sigma+\pi)^{n} d \sigma+\pi^{n} \\
& \ll(\log (n+2))^{n}\left(c_{32} j+1\right)^{j} .
\end{aligned}
$$

Now from this and (4.7), we have

$$
\begin{aligned}
\left|\lambda_{j, l}\right| & \ll \sum_{k=l}^{j} \varrho_{0}^{-j} \frac{\lambda^{k}}{k !} \sum_{i=0}^{k-l}\left(\begin{array}{c}
k \\
i
\end{array}\right)\left(\begin{array}{c}
k-i \\
l
\end{array}\right)(\log \theta)^{k-l-i}(\log (i+2))^{i}\left(c_{32} j+1\right)^{j} \\
& \ll \frac{\left(c_{32} j+1\right)^{j}}{l !} \sum_{k=l}^{j} \frac{\lambda^{k}}{(k-l) !} \sum_{i=0}^{k-l}\left(\begin{array}{c}
k-l \\
i
\end{array}\right)(\log \theta)^{k-l-i}(\log (i+2))^{i} \\
& \ll \frac{\lambda^{l}\left(c_{32} j+1\right)^{j}}{l !} \sum_{k=l}^{j} \frac{\lambda^{k-l}}{(k-l) !}(\log \theta+\log (k-l+2))^{k-l} \\
& \ll \frac{\lambda^{l}\left(c_{32} j+1\right)^{j}(j+2)^{\lambda}}{l !} \ll \frac{\lambda^{l}\left(c_{4} j+1\right)^{j}}{l !} .
\end{aligned}
$$

This completes the proof.

5. Proofs of Theorems 2 and 3. We only prove Theorem 2. The other one can be treated completely in the same way.

It is easy to show that the assumption $(3)^{\prime}$ and $|z| \leq\left((2-\varepsilon) / \psi_{1}\right)^{\psi_{2}}$ imply that $f(n) z^{\Omega(n)}, g(n)$ satisfy the assumption (3) with $\psi=2 \psi_{2}>1$. Thus Theorem 1 allows us to deduce that, uniformly for $|z| \leq\left((2-\varepsilon) / \psi_{1}\right)^{\psi_{2}}$,

$$
\sum_{g(n) \leq x} f(n) z^{\Omega(n)}=\frac{x^{1 / \theta}}{(\log x)^{1-\kappa z / \alpha^{1 / \theta}}}\left\{\sum_{j=0}^{J} \frac{P_{j}\left(\log _{2} x\right)}{(\log x)^{j}}+O\left(R_{J, \lambda}(x)\right)\right\}
$$

where $P_{j}(t):=\sum_{l=0}^{j} \lambda_{j, l}(z) t^{l}$ and the coefficient $\lambda_{j, l}(z)=\lambda_{j, l}\left(f z^{\Omega}, g\right)$ is given by

$$
\lambda_{j, l}(z):=\frac{\theta^{-\kappa z / \alpha^{1 / \theta}}}{l !} \sum_{m=l}^{j} \sum_{k=l}^{m} \sum_{i=0}^{k-l} \lambda_{m, k, i}^{*}(z)
$$

where

$$
\lambda_{m, k, i}^{*}(z):=\frac{(-\log \alpha)^{m}\left(\kappa z \alpha^{-1 / \theta}\right)^{k}(-\log \theta)^{k-l-i} a_{j-m}\left(f z^{\Omega}, g\right) b_{k, m-k}}{\Gamma_{i}\left(\kappa z / \alpha^{1 / \theta}-j\right)(k-l-i) ! i !} .
$$


Obviously $\lambda_{j, l}(z)$ is analytic in the disc $|z|<\left((2-\varepsilon) / \psi_{1}\right)^{\psi_{2}}$ and we can write, in this disc and for any $0<r<\left((2-\varepsilon) / \psi_{1}\right)^{\psi_{2}}$,

$$
\lambda_{j, l}(z)=\sum_{n=0}^{\infty} \chi_{j, l}(n) z^{n}, \quad \chi_{j, l}(n)=\frac{1}{2 \pi i} \int_{|z|=r} \frac{\lambda_{j, l}(z)}{z^{n+1}} d z .
$$

Thus

$$
\begin{aligned}
(\log x)^{\kappa z / \alpha^{1 / \theta}} \lambda_{j, l}(z) & \left(\log _{2} x\right)^{l} \\
= & \left(\log _{2} x\right)^{l} \sum_{m=0}^{\infty} \frac{\left(\kappa \alpha^{-1 / \theta} \log _{2} x\right)^{m}}{m !} \sum_{n=0}^{\infty} \chi_{j, l}(n) z^{m+n} \\
& =\sum_{k=0}^{\infty} z^{k} \sum_{m=0}^{k} \frac{\chi_{j, l}(k-m)\left(\kappa \alpha^{-1 / \theta}\right)^{m}}{m !}\left(\log _{2} x\right)^{l+m} .
\end{aligned}
$$

Notice that

$$
\sum_{\substack{g(n) \leq x \\ \Omega(n)=k}} f(n)=\frac{1}{2 \pi i} \int_{|z|=r} \sum_{g(n) \leq x} f(n) z^{\Omega(n)} \frac{d z}{z^{k+1}},
$$

hence the contribution of the main term in (5.1) is

$$
\begin{aligned}
\frac{x^{1 / \theta}}{\log x} \sum_{j=0}^{J} \frac{1}{(\log x)^{j}} \sum_{l=0}^{j} \sum_{m=0}^{k} \frac{\chi_{j, l}(k-m)\left(\kappa \alpha^{-1 / \theta}\right)^{m}}{m !}\left(\log _{2} x\right)^{l+m} \\
=\frac{x^{1 / \theta}}{\log x} \sum_{j=0}^{J} \frac{Q_{j, k}\left(\log _{2} x\right)}{(\log x)^{j}} .
\end{aligned}
$$

It remains to estimate the error term. Taking $r=k / \log _{2} x$ and writing $\kappa=|\kappa| e^{i \phi}$ show that this is

$$
\begin{aligned}
& \ll_{A} \frac{x^{1 / \theta} R_{J, \lambda}(x)}{\log x} \oint_{|z|=r} \frac{(\log x)^{\operatorname{Re}\left(\kappa z / \alpha^{1 / \theta}\right)}}{|z|^{k+1}}|d z| \\
& \ll_{A} \frac{x^{1 / \theta} R_{J, \lambda}(x)}{\log x}\left(\frac{\log _{2} x}{k}\right) \int_{0}^{k} e^{2 \pi|\kappa| \alpha^{-1 / \theta} \cos (\phi+\vartheta)} d \vartheta .
\end{aligned}
$$

Denote the last integral by $I$. Then

$$
\begin{aligned}
I & \leq 4 \int_{0}^{\pi / 2} e^{k|\kappa| \alpha^{-1 / \theta} \cos \vartheta} d \vartheta=4 \int_{0}^{1} \frac{e^{k|\kappa| \alpha^{-1 / \theta} t}}{\sqrt{1-t^{2}}} d t \\
& \leq 4 e^{k|\kappa| \alpha^{-1 / \theta}} \int_{0}^{1} \frac{e^{-k|\kappa| \alpha^{-1 / \theta}}(1-t)}{\sqrt{1-t}} d t \ll \frac{e^{k|\kappa| \alpha^{-1 / \theta}}}{\sqrt{|\kappa| k+1}} .
\end{aligned}
$$

This completes the proof. 
6. Proofs of Corollaries 2 and 3. As before, we only prove Corollary 2 and the other one will follow by the same argument.

We choose $\varepsilon=\varepsilon(\delta)>0$ so small that $\{(2-\varepsilon) /(1 / 2+10 \varepsilon)\}^{1 / 2+\varepsilon}>2-\delta$. It is easy to verify that $f(n) \equiv 1$ and $\varphi(n)$ satisfy the assumptions (1)-(3)' with $\psi_{1}=1 / 2+10 \varepsilon$ and $\psi_{2}=1 / 2+\varepsilon$. Thus Theorem 2 is applicable. Next we show that the main term can take the simple form as stated. In view of Remarks (ii), we have $\lambda_{j, l}(z)=0$ for $l=1, \ldots, j$ and $\lambda_{j, 0}(z)=a_{j}\left(z^{\Omega}, \varphi\right) / \Gamma(z-j)$. Observing that $\lambda_{j, 0}(0)=0$, we see that $\varsigma_{j}(z):=a_{j}\left(z^{\Omega}, \varphi\right) /(z \Gamma(z-j))$ is analytic in the disc $|z| \leq 2-\delta$. Thus Theorem 2 implies (1.14).

Now we prove (1.15). If $k=1$, the formula (1.15) can be obtained directly from Theorem 2 with $J=0$. Next suppose $k \geq 2$. Similarly to (5.1) with $J=0$, we have, uniformly for $|z| \leq 2-\delta$,

$$
\sum_{\varphi(n) \leq x} z^{\Omega(n)}=\frac{x}{(\log x)^{1-z}}\left\{z \varsigma_{0}(z)+O\left(\frac{1}{\log x}\right)\right\} .
$$

Dividing both sides by $2 \pi i z^{k+1}$ and integrating over $|z|=r$ yields, for any $r \leq 2-\delta$,

$$
\sum_{\substack{\varphi(n) \leq x \\ \Omega(n)=k}} 1=\frac{x}{\log x} \cdot \frac{1}{2 \pi i} \int_{|z|=r} \frac{\varsigma_{0}(z)(\log x)^{z}}{z^{k}} d z+O_{\delta}(R),
$$

where

$$
R:=\frac{x}{(\log x)^{2}} \int_{|z|=r} \frac{(\log x)^{\operatorname{Re} z}}{|z|^{k+1}}|d z| .
$$

To evaluate the principal term in (6.1), we write

$$
\varsigma_{0}(z)=\varsigma_{0}(r)+(z-r) \varsigma_{0}^{\prime}(r)+(z-r)^{2} \int_{0}^{1}(1-t) \varsigma_{0}^{\prime \prime}(r+t(z-r)) d t .
$$

Taking $r:=(k-1) / \log _{2} x$, the Cauchy formula gives

$$
\frac{1}{2 \pi i} \int_{|z|=r} \frac{(z-r)(\log x)^{z}}{z^{k}} d z=\frac{\left(\log _{2} x\right)^{k-2}}{(k-2) !}-r \frac{\left(\log _{2} x\right)^{k-1}}{(k-1) !}=0 .
$$

Therefore it follows that

$$
\begin{aligned}
\frac{1}{2 \pi i} \int_{|z|=r} \frac{\varsigma_{0}(z)(\log x)^{z}}{z^{k}} d z & =\frac{1}{2 \pi i} \int_{|z|=r} \frac{\varsigma_{0}(r)(\log x)^{z}}{z^{k}} d z+O_{\delta}\left(R^{\prime}\right) \\
& =\frac{\left(\log _{2} x\right)^{k-1}}{(k-1) !} \varsigma_{0}\left(\frac{k-1}{\log _{2} x}\right)+O_{\delta}\left(R^{\prime}\right)
\end{aligned}
$$


where

$$
R^{\prime}:=\int_{|z|=r}\left|(z-r)^{2} \int_{0}^{1}(1-t) \varsigma_{0}^{\prime \prime}(r+t(z-r)) d t\right|(\log x)^{\operatorname{Re} z}|z|^{-k}|d z| .
$$

Since $|r+t(z-r)| \leq(1-t) r+t r=r(0 \leq t \leq 1,|z|=r)$, we have $\varsigma_{0}^{\prime \prime}(r+t(z-r)) \ll_{\delta} 1$ and

$$
\begin{aligned}
R^{\prime} & \ll_{\delta} \int_{0}^{2 \pi}\left|e^{i \vartheta}-1\right|^{2} r^{3-k} e^{r \log _{2} x \cos \vartheta} d \vartheta \\
& \ll_{\delta} r^{3-k} \int_{0}^{2 \pi} e^{(k-1) \cos \vartheta}(1-\cos \vartheta) d \vartheta \\
& \ll_{\delta} r^{3-k}\left(\int_{0}^{1} e^{(k-1) t} \sqrt{1-t} d t+2 \pi\right) \ll_{\delta} r^{3-k} e^{k-1}(k-1)^{-3 / 2} \\
& \ll_{\delta} \frac{\left(\log _{2} x\right)^{k-1}}{(k-1) !} \cdot \frac{k-1}{\left(\log _{2} x\right)^{2}} .
\end{aligned}
$$

Similarly, we have

$$
R \ll_{\delta} \frac{x}{(\log x)^{2}} e^{k-1} r^{-k}(k-1)^{-1 / 2} \ll_{\delta} \frac{x}{\log x} \cdot \frac{\left(\log _{2} x\right)^{k-1}}{(k-1) !} \cdot \frac{k-1}{\left(\log _{2} x\right)^{2}}
$$

The estimate (1.15) now follows from (6.2)-(6.4). This completes the proof.

\section{References}

[1] R. Balasubramanian and K. Ramachandra, On the number of integers $n$ such that $n d(n) \leq x$, Acta Arith. 49 (1988), 313-322.

[2] M. Balazard et G. Tenenbaum, Sur la répartition des valeurs de la fonction d'Euler, Compositio Math. 110 (1998), 239-250.

[3] P. T. Bateman, The distribution of values of the Euler function, Acta Arith. 21 (1972), 329-345.

[4] H. Davenport, Multiplicative Number Theory, 2nd ed., Springer, New York, 1980.

[5] P. Erdős, Some remarks on Euler's $\varphi$-function and some related problems, Bull. Amer. Math. Soc. 51 (1945), 540-544.

[6] S. W. Graham and G. Kolesnik, Van der Corput's Method of Exponential Sums, Cambridge Univ. Press, 1991.

[7] E. Scourfield, On some sums involving the largest prime divisor of $n$, Acta Arith. 59 (1991), 339-363.

[8] A. Selberg, Note on the paper by L. G. Sathe, J. Indian Math. Soc. 18 (1954), 83-87.

[9] A. Smati, Une formule asymptotique pour une classe de fonctions multiplicatives, Publ. Inst. Math. (Beograd) (N.S.) 49 (1991), 83-91.

[10] - Répartition des valeurs d'une classe de fonctions multiplicatives, Acta Arith. 61 (1992), 83-100. 
[11] A. Smati and J. Wu, Distribution of values of some multiplicative functions over integers free of large prime factors, Quart. J. Math. Oxford (2) 50 (1999), 111-130.

[12] G. Tenenbaum, Introduction to Analytic and Probabilistic Number Theory, Cambridge Univ. Press, 1995.

Institut Élie Cartan

UMR 7502 UHP-CNRS-INRIA

Université Henri Poincaré (Nancy 1)

54506 Vandœuvre-lès-Nancy, France

E-mail: lau@iecn.u-nancy.fr

wujie@iecn.u-nancy.fr

Received on 23.10.2000

and in revised form on 30.4.2001 